Preprints of the

Max Planck Institute for

Research on Collective Goods

Bonn 2008/12

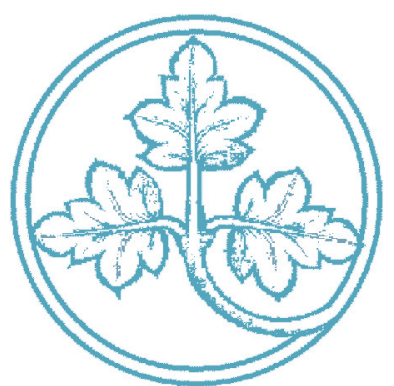

Multiple-Reason Decision Making Based on Automatic Processing

Andreas Glöckner / Tilmann Betsch

This paper can be downloaded free of charge from the Social Science Research Network at http://ssrn.com/abstract=1133445

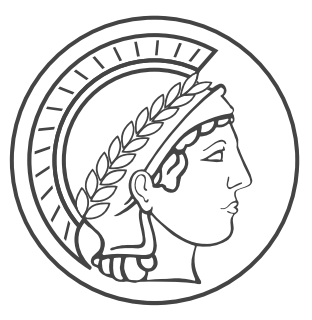




\section{Multiple-Reason Decision Making Based on Automatic Processing}

Andreas Glöckner / Tilmann Betsch

April 2008 


\title{
Multiple-Reason Decision Making Based on Automatic Processing*
}

\author{
Andreas Glöckner / Tilmann Betsch
}

\begin{abstract}
It has been repeatedly shown that in decisions under time constraints, individuals predominantly use noncompensatory strategies rather than complex compensatory ones. We argue that these findings might be due not to limitations of cognitive capacity but instead to limitations of information search imposed by the commonly used experimental tool Mouselab (Payne et al., 1988). We tested this assumption in three experiments. In the first experiment, information was openly presented, whereas in the second experiment the standard Mouselab program was used under different time limits. The results indicate that individuals are able to compute weighted additive decision strategies extremely quickly if information search is not restricted by the experimental procedure. In a third experiment, these results were replicated using more complex decision tasks, and the major alternative explanations that individuals use more complex heuristics or merely encode the constellation of cues were ruled out. In sum, the findings challenge the fundaments of bounded rationality and highlight the importance of automatic processes in decision making.
\end{abstract}

Keywords: Automatic Information Integration, One Reason Decision Making, Mouselab, Probabilistic Inferences, Process Tracing, Time Limits, Intuition

Andreas Glöckner, Max Planck Institute for Research on Collective Goods, Bonn and Tilmann Betsch, University of Erfurt, Germany. The first two studies were conducted as a part of the diploma thesis of the first author at the University of Heidelberg, Germany. We thank Stephan Dickert, Christoph Engel, Martin Beckenkamp, Peter Ayton, Edward Wisniewski and three anonymous reviewers for helpful comments on earlier drafts of this article which helped to improve it considerably. Correspondence concerning this article should be addressed to Andreas Glöckner, Max Planck Institute for Research on Collective Goods, Kurt-Schumacher-Str. 10, D-53113 Bonn, Germany. E-Mail: gloeckner@coll.mpg.de. 


\section{Multiple-Reason Decision Making Based on Automatic Processing}

In process tracing studies in decision research, evidence has accumulated that individuals often employ simple strategies that minimize the amount of information considered and the mental effort invested in a decision (e.g., Payne, Bettman, \& Johnson, 1988). These strategies, such as the lexicographic rule ( $L E X$, Fishburn, 1974), elimination-by-aspects ( $E B A$, Tversky, $1972)$, or the equal weight rule (EQW, Fishburn, 1974) involve considerably less computational steps than the weighted additive rule $(W A D D)$ of utility theory. Scholars now take it for granted that time and capacity constraints provoke strategy shifts from complex, compensatory strategies towards simple noncompensatory ones (Ariely \& Zakay, 2001; Bettman, Luce, \& Payne, 1998; Payne et al., 1988; Payne, Bettman, \& Johnson, 1992; Rieskamp \& Hoffrage, 1999). In this paper we question the external validity of the findings underlying this conclusion. Specifically, we argue that the predominantly used research methods in behavioral decision research encourage participants to deliberate and hinder the activation of automatic decision-making processes. We aim to demonstrate that automatic processes driven by the "intuitive system" (Kahneman \& Frederick, 2002) enable individuals to quickly integrate multiple reasons in their decisions in a compensatory way. In two of the three experiments reported in this paper, we applied a research method in which the intuitive system could demonstrate its powers. Results indicate that the majority of individuals use automatic processes to integrate multiple reasons in a weighted additive manner if information acquisition is not constrained by the experimental setting.

\section{Bounded Rationality and the Focus on Deliberate Decision Strategies}

In line with the dual-processing framework suggested by Kahneman and Frederick (2002), we define deliberate decision strategies as strategies that are based on controlled cognitive operations. Here, information is integrated in a serial manner, processing is cognitively demanding and rather slow, and individuals using these strategies are aware of most of the underlying processes and can even verbalize them. Research in the tradition of the bounded rationality approach (Simon, 1955) has focused on the functioning of such deliberate decision strategies (cf. Frederick, 2002). The fundamental tenet of the bounded rationality approach is that, because of their limited cognitive capacities, individuals employ shortcut strategies (commonly called heuristics ${ }^{l}$ ).

A prominent decision problem that has been repeatedly used in this research is the city-size decision task (Gigerenzer, Hoffrage, \& Kleinbölting, 1991; Gigerenzer, Todd, \& the ABC Group, 1999). In this task, an individual has to decide which of two cities (options) has more inhabitants based on conflicting pieces of evidence (cues). Cues (e.g., whether a city is a state capital) vary as predictors for city size, which means that they differ in the conditional likeli-

1 Note that in research on human judgments, the term heuristics refers to strategies that are based on automatic processes (cf. Kahneman \& Frederick, 2002). 
hood (cue validity) that city $\mathrm{A}$ is larger than city $\mathrm{B}$ given a positive cue value. Assume that the individual is informed by the experimenter that city $\mathrm{A}$ is a state capital and city B is not, and that city B has a university and a major league sports team whereas city A has neither. A very simple strategy would be to base the decision on only the most valid cue. If, for instance, the person presumes that the state capital cue is the most valid one, he or she could consider only this information, ignore the other cues, and decide that city A is larger. Such a strategy relies on one reason only. This example describes the application of the Take-The-Best heuristic (TTB, Gigerenzer \& Goldstein, 1996), which belongs to the class of LEX rules. Only if the most valid cue does not differentiate between options would the next cue be used, and so on. Such a strategy seems to be simple enough for laypersons to apply deliberately in everyday settings.

A strategy in which all the validities of all cue values are considered would be much more complicated. This could be realized by a weighted additive rule (WADD) in which cue values are multiplied by the validities of the respective cues and summed up. The option with the highest weighted sum (total evidence) is chosen. WADD considers all relevant pieces of information, integrates cue values and their validities for each option, and thus provides an ideal example of a compensatory strategy (i.e., a strategy in which negative values on one cue can be compensated for by positive values on other cues). It has been repeatedly stated by proponents of the bounded rationality approach that humans might be often incapable of applying such extensional strategies because these strategies overtax their computational capacities (e.g., Gigerenzer et al., 1999).

As will be pointed out in more detail later, we question this assumption and argue that individuals may indeed carry out a WADD strategy, albeit not by deliberately calculating weighted sums but instead by relying on automatic processes.

\section{Mouselab - A Method for Process Tracing and Strategy Classification}

One of the major challenges in behavioral decision research is to empirically identify application of different decision strategies on the individual level, as the underlying cognitive processes cannot be directly observed. Hence, a multitude of methods have been developed to infer decision strategies from proximal parameters such as choice patterns (e.g., Bröder \& Schiffer, 2003b), information search parameters (e.g., Johnson, Payne, Schkade, \& Bettman, 1986; Sundstroem, 1987), decision times (e.g., Bergert \& Nosofsky, 2007; Glöckner, 2006; Glöckner, 2007), confidence judgments (Glöckner \& Hodges, 2006), eye movements (e.g., Russo \& Dosher, 1983), self-reports and think-aloud protocols based on introspection (e.g., Montgomery \& Svenson, 1983; Svenson, 1989), or combinations of these.

One of the standard tools for strategy classification is the computer-based information board called Mouselab (Johnson et al., 1986). In Mouselab, information about options is presented in a covered information matrix. Participants have to move the mouse cursor onto boxes to 
uncover the outcomes of choice options. Steps of information search are recorded and are subsequently used to identify decision strategies (see Figure 3 for an example).

The introduction of Mouselab was an important breakthrough. By providing an easy-tohandle tool for process tracing and strategy classification, this research method opened the door to a process view in decision research; hence, it might actually be considered a revolution (Beach \& Potter, 1992). Regardless of its undisputed merits, the method entails some difficulties. The fundamental problem is that it imposes restrictions on information search that might, in turn, influence strategy selection. Note, for instance, that in the standard Mouselab program, only one piece of information can be inspected at a time. This procedure promotes a serial mode of information search and hampers the possibility of making quick comparisons between multiple pieces of information as well as of detecting specific cue constellations. Thus, Mouselab fosters application of deliberate, step-by-step decision strategies and hinders the activation of automatic processes. Therefore, findings from Mouselab studies are likely to underestimate humans' total cognitive capacity, which is based on the usage of both types of processes. Furthermore, Mouselab confounds the sources of constraints. Accordingly, the use of simple strategies may reflect both the constraints on overt search behavior imposed by the properties of the research tool and the constraints on the processing system imposed by capacity limitations of the cognitive system.

In Mouselab experiments, it has been consistently observed that participants change decision strategies under severe time pressure from more complex, compensatory strategies to simple, noncompensatory ones (e.g., from WADD to LEX; Payne et al., 1988; Rieskamp \& Hoffrage, 1999; for further discussions, see Ariely \& Zakay, 2001; Broadbent, 1971; Zakay, 1993). We do not want to claim that these findings are always and entirely produced by Mouselab, particularly because there is converging evidence from studies which used other methods (Edland \& Svenson, 1993). However, we would like to highlight the problem that these strategy shifts may only apply to deliberate decision strategies. Mouselab is likely to support the application of these strategies and to hamper the application of automatic processes. The relatively high decision times usually observed in Mouselab experiments clearly indicate that decision strategies are based on elaborated deliberation (e.g., the average time for individuals' decisions was for instance 44 seconds in Payne et al., 1988, Experiment 1, no time pressure condition). Thus, a critical scrutiny of the procedure reveals that the results cannot serve as conclusive evidence for the view that the strategy shift is always caused by a limited capacity for information integration. Neither can they rule out the alternative explanation that time pressure simply constrains the information search operations (i.e., movements of the computer mouse) necessary for gaining access to the information and thus also hampers automatic decision processes.

Findings by Lohse and Johnson (1996) lend support for this alternative interpretation. The authors investigated the influence of different types of process tracing methods on decision behavior and identified, apart from a significant amount of convergence, substantial differences between information search behavior and choices when the same decision tasks were 
presented in Mouselab, where information had to be looked up serially, or presented openly so that information was instantly accessible; in the latter case, information search was recorded using an eye tracking method. Lohse and Johnson found that the Mouselab method significantly increased the amount of time needed to acquire information compared with the second method. In the Mouselab condition, individuals also showed a more systematic information acquisition behavior. Finally, in the condition containing richer contextual information (i.e., decisions between apartments as compared to decisions between gambles), almost one third of the individual choices changed as a function of the manipulation of the process tracing method (see also Billings \& Marcus, 1983; Maule, 1994).

Taken together, findings indicate that Mouselab forces decision makers to engage in a serial consideration of information. In turn, this method induces a deliberate rule-based integration of information. These processes are slow and consume both task and mental resources. Automatic processes that could draw on a parallel consideration of information are systematically constrained in this paradigm. Unsurprisingly, individuals reduce the depth of serial processing in Mouselab, especially when time and cognitive resources become scarce. When time pressure conditions do not allow all information to be inspected, the Mouselab method invites the application of noncompensatory strategies (e.g., LEX/TTB). We cannot rule out the possibility that the presumed increased prevalence of noncompensatory strategies under time pressure mainly applies to situations that resemble Mouselab. Thus, the general claim that individuals always use this kind of strategy more often under time pressure is not warranted.

\section{The Neglected Role of Automatic Processes in Research on Decision Strategies}

Although the importance of automatic processes has been repeatedly highlighted (Bargh \& Chartrand, 1999; Bargh \& Williams, 2006; Doherty \& Kurz, 1996; Hasher \& Zacks, 1984; Hintzman, 1988; Kahneman, Slovic, \& Tversky, 1982; Kahneman \& Frederick, 2002; Schneider \& Shiffrin, 1977; Shiffrin \& Schneider, 1977; Wegner, 1994; Zajonc, 1980), they were largely ignored in research on multiple-strategy decision making (Frederick, 2002). Elaborating on the notion of a dual-processing approach (see Chaiken \& Trope, 1999, for an overview), Kahneman and Frederick (2002) put forward a two-system framework that distinguishes between intuitive/automatic processes (system 1) and reflective/deliberate processes (system 2). It is usually assumed that shortcut strategies like EQW or LEX/TTB are executed by the deliberate system since they draw upon controlled processes.

Beyond this general framework, several models have been proposed that specify the functioning of automatic processes (e.g., Beach \& Mitchell, 1996; Betsch, 2007; Busemeyer \& Townsend, 1993; Dougherty, Gettys, \& Ogden, 1999; Epstein, 1990; Frederick, 2002; Glöckner, 2006; Glöckner \& Betsch, 2008; Hogarth, 2001; Lieberman, 2000; Simon, Snow, \& Read, 2004; Sloman, 2002). It is beyond the scope of this paper to discuss and compare these models. For simplicity, we base our research on one fundamental assumption raised by Hammond et al., (1987): 
General Hypothesis: Automatic processes should enable individuals to quickly integrate information in a weighted additive manner.

This would mean that individuals apply a WADD rule without deliberately calculating weighted sums.

\section{Methodological Preliminaries}

Notation. We use the abbreviation $W A D D$ to refer to a strategy that integrates cue values and cue validities in weighted additive (linear) fashion. On a general level, we use WADD in a paramorphic sense (Hoffman, 1960). Accordingly, WADD is said to be applied if the output of a decision (the choice) accords to the choice predictions derived from a linear aggregation of all the given pieces of information available. If we wish to address the level of processes (i.e., what individuals really do when they make a decision), we add suffixes specifying the type of processes. Specifically, we use the notation $W A D D_{d e l}$ for a strategy that is based on a deliberate calculation of weighted sums and the notation $W A D D_{\text {auto }}$ for a strategy that performs the integration operations automatically. LEX/TTB describes noncompensatory onereason decision strategies that search cues in the order given by cue validities. EQW describes equal weight strategies in which cue validities are ignored and the option with more positive cue values is selected.

Analysis of Choices in Diagnostic Decision Tasks. Strategy classification in our experiments is primarily based on the analysis of choices. Decision tasks were systematically selected as diagnostic for different decision strategies (cf. Glöckner \& Betsch, in press). Specifically, decision tasks were based on cue patterns (constellations of cue information) so that the considered strategies LEX/TTB, EQW, and WADD make different predictions for substantial subsets of tasks. To allow for a classification of decision strategies on an individual level as compared to an analysis across all participants, decision tasks were presented repeatedly by holding constant the structural cue patterns underlying the decision tasks (cf. Bröder \& Schiffer, $2003 \mathrm{~b}$ ). Note that it is obviously impossible to differentiate between $\mathrm{WADD}_{\text {del }}$ and $\mathrm{WADD}_{\text {auto }}$ based on choices because choice predictions are equal; therefore, we have to consider other process-related variables, such as decision time.

Analysis of Decision Time Patterns. The process of information integration will be further investigated by analyzing individual decision time patterns (cf. Bergert \& Nosofsky, 2007). Strategies differ considerably with respect to the time their performance expends. For individuals who use a LEX/TTB strategy, decision times should depend on the number of cues required for differentiating between the options. Thus, people should decide faster in decision tasks in which the first cue differentiates between options as compared to decision tasks in which two or more cues have to be considered (Bröder \& Gaissmaier, in press). Individuals who use an EQW strategy should not show any differences in decision times as long as the number of cue values is held constant and the sum of cue values differentiates between op- 
tions. The same prediction holds for a $\mathrm{WADD}_{\text {del }}$ strategy that is based on a deliberate calculation of weighted sums (Payne et al., 1988). In contrast, some of the decision strategies based on automatic processes (e.g., $\mathrm{WADD}_{\text {auto }}$ ) facilitate deriving the prediction that decision times increase with rising evidence that points against the preferred option, and decrease with rising evidence in favor of the preferred option (Busemeyer \& Townsend, 1993; Cartwright \& Festinger, 1943; Glöckner, 2006; cf. Holyoak \& Simon, 1999; for empirical evidence in favor of this claim, see Festinger, 1943; Glöckner, 2007). The general version of the rational model (Bergert \& Nosofsky, 2007) specifically predicts that decision time decreases with increasing difference between the total evidence for two options. Furthermore, it can be predicted that the overall level of decision time is much higher for a $\mathrm{WADD}_{\text {del }}$ strategy than a $\mathrm{WADD}_{\text {auto }}$ strategy. Thus, besides providing converging evidence for the choice-based strategy classification method, decision time analysis can be used to test whether individuals applied a $\mathrm{WADD}_{\text {del }}$ or a $\mathrm{WADD}_{\text {auto }}$ strategy. In our experiments, decision times were analyzed to further differentiate between decision strategies and to learn more about the underlying processes.

Analysis of Confidence Judgments. Other data that depend on the applied decision strategies and thus could be used to learn more about the processes of decision making are subjective judgments of the confidence in choices (Christensen-Szalanski, 1978). According to simple LEX/TTB rules, confidence should depend on the validity of the most valid cue only (Gigerenzer et al., 1991), whereas some of the WADD models predict that confidence is dependent on the differences in the weighted cue values for the options (Cartwright \& Festinger, 1943; Glöckner, 2006). Bergert and Nosofsky (2007) refer to decisions with a low (vs. high) difference in the total evidence for the options as hard (vs. easy) decisions. Assuming that hard decisions lead to lower confidence judgments than easy decisions do, the same prediction could be derived from Bergert and Nosofsky's generalized version of the rational model. In the third experiment, we therefore investigated confidence judgments. Table 1 summarizes the differential predictions of the decision strategies that were used to identify strategies in the three experiments reported in this paper.

In the first experiment reported below, we tested whether individuals are able to quickly integrate information in a weighted additive manner (application of a $\mathrm{WADD}_{\text {auto }}$ strategy) if information search is not restricted by the research tool. In the second experiment, the decision tasks of Experiment 1 were presented in a classical Mouselab format under different time limit conditions in order to further investigate whether strategy shifts are due to limitations of cognitive capacity or to limitations of information search induced by the Mouselab method itself. In the third experiment, more complex decision tasks and a manipulation of cue validities were used that enabled further investigation of information integration processes. 


\begin{tabular}{|c|c|c|c|c|}
\hline & \multicolumn{4}{|c|}{ Decision Strategies } \\
\hline & LEX/TTB & EQW & $W^{\prime} A D D_{\text {del }}$ & WADD auto \\
\hline \multicolumn{5}{|c|}{ Choices } \\
\hline 1. Less valid cues are ignored ${ }^{1,2,3}$ & Yes & No & No & No \\
\hline 2. Cue validities are ignored $d^{1,2,3}$ & No & Yes & No & No \\
\hline 3. Weighted additive information integration $n^{1,2,3}$ & No & No & Yes & Yes \\
\hline \multicolumn{5}{|c|}{ Decision Times } \\
\hline $\begin{array}{l}\text { 1. Time dependent on the cues necessary for dif- } \\
\text { ferentiating with a LEX strategy }{ }^{1}\end{array}$ & Yes & No & No & No \\
\hline 2. Time equal for all cue patterns ${ }^{1,3}$ & No & Yes & Yes & No \\
\hline $\begin{array}{l}\text { 3. Time decreases with increasing differences } \\
\text { between the options }{ }^{1,3}\end{array}$ & No & No & No & Yes \\
\hline \multicolumn{5}{|c|}{ Confidence Judgments } \\
\hline $\begin{array}{l}\text { 1. Confidence dependent on the validity of the } \\
\text { differentiating cue with LEX }\end{array}$ & Yes & No & No & No \\
\hline $\begin{array}{l}\text { 2. Confidence increases with increasing differ- } \\
\text { ences between the options }{ }^{3}\end{array}$ & No & No & Yes & Yes \\
\hline
\end{tabular}

Note. Hypotheses are stated in the left-hand column. Exponents indicate the experiment(s) in which each hypothesis was tested. The predictions of the decision strategies are presented in the columns to the right with the values yes-no indicating that the respective hypothesis should or should not hold if the strategy is applied.

\section{Experiment 1}

In Experiment 1 information was presented in an "open" matrix (no covered information) to assure that the information search was not artificially constrained. All participants were put under time pressure by the instructions. According to the bounded rationality approach, individuals should use simple deliberate strategies (i.e., LEX/TTB) under such conditions because they are assumed to lack the cognitive capacity to perform complex calculations when decision time is limited. The alternative hypothesis states that even under time pressure, participants use a $\mathrm{WADD}_{\text {auto }}$ strategy because the automatic system is able to simultaneously process a multitude of information (e.g., in a parallel and holistic fashion, Glöckner \& Betsch, 2008).

The experimental tasks required the participants to assume the role of a manager of a company. In repeated decision trials, participants were instructed to select the best of three different products (options). They were provided with information from three testers (cues) with different predictive validity (cue validity), which provided dichotomous quality ratings (i.e., good-bad) for each product. 


\section{Method}

Participants and design. Participants in the first experiment were 15 University of Heidelberg students (11 female, 4 male). The experiment lasted approximately 30 minutes. Participation was either compensated for by course credit or a flat fee amounting to 4 euros. Decision tasks were varied as a within-participants factor, resulting in a 6 (VERSION) x 23 (CUE PATTERN) design with the following factors nested under the latter: CUE (number of cues necessary to differentiate according to a LEX/TTB rule), PRO_OPTION1 (number of positive cue values in favor of option 1), and PRO_OPTION2/3 (number of positive cue values in favor of option 2 or 3). The factor VERSION represented six different versions of each cue pattern, in which the order of options was permutated. Table 2 shows the 23 cue patterns used in the experiment. $\mathrm{C} 1$ to $\mathrm{C} 3$ refer to cues in order of validity with cue 1 being the most valid cue. The cue validities (in this case given as probabilities of correct predictions) were .80, .70, and .50. $\mathrm{O} 1$ to $\mathrm{O} 3$ represent the eligible options. Cue values are represented by the symbols "+" (positive) and "-" (negative).

The 23 cue patterns can be separated into three sets that correspond to the manipulation of the factors CUE. In the first set (CUE PATTERNS 1 to 15; CUE=1), the most valid cue has only one positive cue value in favor of option 1 . Within set 1 , the number of cues with a positive cue value for option 1 (PRO_OPTION1) varies from 1 to 3 (cf. main rows in Table 2). This variation is completely crossed with a variation of the number of positive cue values for options 2 and 3 (PRO_OPTION2/3) from 0 to 4 (cf. main columns in Table 2), resulting in a total of 15 stimuli for set 1 . In the second set (CUE PATTERNS 16 to 21 ; CUE=2), cue 1 has more than one positive cue value but cue 2 has only one. In the third set (CUE PATTERNS 22 and 23; $C U E=3$ ), cue 1 has entirely positive cue values, cue 2 has two positive cue values, and cue 3 has one or two.

Cue Patterns and Strategy Classification. Individuals who use a LEX/TTB strategy should show increasing decision times from set 1 to set 3 but constant decision times within each given set because a constant number of cues need to be considered to arrive at a decision. Individuals who use a $\mathrm{WADD}_{\text {auto }}$ strategy should show increasing decision times with increasing evidence for options 2 and 3 (PRO_OPTION2/3) and decreasing decision times with increasing evidence for option 1 (PRO_OPTION1). Individuals that use a WADD del rule should show equal decision times for all cue patterns. The 23 cue patterns also allowed for classification of the decision strategies LEX/TTB, EQW and WADD based solely on the choice analysis. The LEX/TTB strategy predicts choices of option 1 in all 23 cue patterns, because the most valid differentiating cue (i.e., cue 1 for cue patterns 1 to 15 ; cue 2 for cue patterns 16 to 21; cue 3 for cue patterns 22 and 23) always points towards this option. The EQW and WADD strategies predict choices of option 2 in cue patterns 7 and 10, and choices of options 2 or 3 in cue pattern 13, because in these patterns both the unweighted and the weighted sum of the cue values are higher for these options. Note that for a WADD strategy, this prediction is only valid if the sum of the subjective cue validities for cues 2 and 3 is higher than the cue validity of cue 1 (see also Footnote 3 below). 
Table 2

Cue Patterns Experiment 1 and 2

Cue Patterns Set 1 (CUE=1)

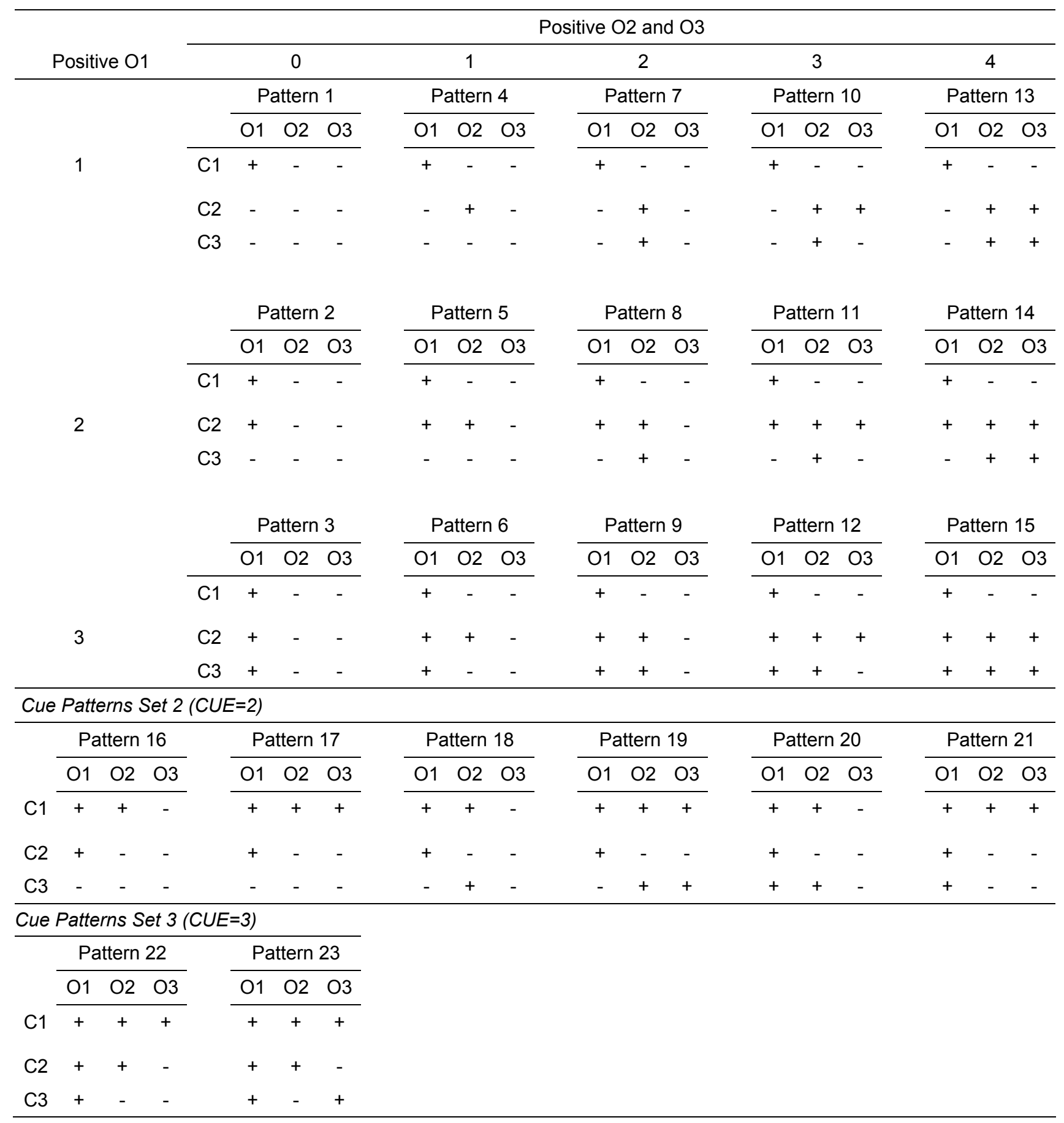

Note. The 23 cue patterns used in Experiment 1 and 2 are depicted in a matrix format. C1 to C3 represent cues 1 to 3 , with cue 1 being the most valid and cue 3 the least valid cue. 01 to 03 represent options. Cue patterns are categorized in three sets for which the number of cues increases. Set 1 consists of cue patterns 1 to 15 , set 2 consists of cue patterns 16 to 21 , and set 3 consists of cue patterns 22 and 23 . Within set 1 , the number of positive cue values for option 1 is varied from 1 to 3 (cf. main rows). This variation is fully crossed with a variation of the number of positive cue values for options 2 and 3 ( 0 to 4 ; cf. main columns). 
Individuals who choose option 1 in all cue patterns can be classified as LEX/TTB users, whereas individuals who mainly choose option 2 or 3 in cue patterns 7, 10, and 13 could have used EQW or WADD. The latter participants were further differentiated based on an examination of cue patterns $4,8,11$, and 18 . The EQW strategy predicts an equal distribution of choices of options 1 and 2 for these patterns because the number of cues is equal for both options, whereas WADD predicts choices of option 1 because the more valid cues speak for this option. In summary, people who choose option 1 in all cue patterns ignore less valid cues and should be classified as LEX/TTB users; individuals who mainly choose option 2 or 3 in cue patterns 7, 10, and 13 and about equally often choose option 1 and 2 in cue patterns 4, 8, 11, and 18 look at all cue values but ignore cue validities, and should be classified as EQW users; and finally, individuals who choose option 2 or 3 in cue patterns 7, 10, and 13 and mainly choose option 1 in cue patterns $4,8,11$, and 18 take into account all the cue values as well as their validities, and should be classified as WADD users (as mentioned above, the notion is used in a paramorphic sense, not implying that weighted sums are calculated in a serial manner).

Materials and Procedure. A computer program written in MEL2 (Multiple Experimental Language 2) was used to run the experiment. The complete experimental instructions can be found in Appendix A. Participants were instructed to repeatedly select the vendor who provides the best quality product. They were informed about the testers' cue validities in a frequency format to facilitate their understanding and processing of the information (Gigerenzer \& Hoffrage, 1995). Note that from a normative perspective, participants should ignore the information of tester 3 (i.e., 50 percent correct predictions) since the validity of this information reaches only the level of chance, which should in turn encourage the application of LEX/TTB strategies. Moreover, participants were asked to make high quality decisions and to be as fast as possible in deciding (Fazio, 1990). All nine pieces of information for each decision task were presented simultaneously in an information matrix with cues displayed in rows and options in columns. Information was presented in the middle of a black screen using ASCII characters as depicted in Appendix A. The information remained on the screen until the participants chose one option by hitting one of three adjacent keys that were marked on the keyboard (i.e., "f", “g”, "h"). Choices and decision times were recorded.

Eight warm-up decision trials were used to familiarize participants with the material and the procedure. These were followed by 138 target trials, which were presented in six randomized presentation blocks, each consisting of one of the six versions of the 23 choice patterns. Two 1-minute breaks were embedded to minimize the effects of decreasing concentration. After having completed all tasks, participants were asked to recall the cue validities in order to ensure that they had remained aware of them over the entire course of the experiment. 


\section{Results}

Strategy Classification Based on Choices. Choice proportions for option 1 in cue patterns 1 to 23 aggregated across VERSIONS are depicted in Figure 1. On an aggregated level of analysis, choices of option 1 were most frequently observed, except in the critical patterns 7,10 , and 13. Thus, the majority of aggregated choices are in line with the predictions of WADD, indicating that a considerable portion of participants used this strategy. In order to determine the size of this portion precisely, further analyses were conducted on the individual level. To identify participants who used a LEX/TTB strategy, choices in cue patterns 7, 10, and 13 were compared with choices in the remaining cue patterns. As mentioned above, in an error-free application of a LEX/TTB strategy, option 1 should always be selected. Taking into account that individuals may not be able to apply a decision strategy without error, one might decide to determine a proper error rate. Given that this is methodologically problematic, however, one can alternatively test whether the observed individual rate of error is the same for different cue patterns (cf. Bröder \& Schiffer, 2003b). Thus, if a LEX/TTB strategy is applied, the portion of choices of option 1 should be equal in the critical cue patterns compared to in the remaining ones. This hypothesis was tested using individual $\chi^{2}$-tests of independence. For each participant, it was tested whether the proportion of choices of option 1 were independent of cue patterns. Here, cue patterns 7, 10, and 13 (critical cue patterns) were compared with the remaining cue patterns. Eleven participants chose option 1 significantly less often in the critical patterns than in the remaining patterns $(p<.05)$. This indicates that the information provided by the less valid cues systematically influenced their choices, even though the most valid cue already discriminates between the options (cf. Table 2). Thus, it is unlikely that these participants used a LEX/TTB strategy. Two of the 15 participants made their choices in line with the predictions of a LEX/TTB strategy and were classified respectively. Two further participants distributed their choices equally among options 1,2, and 3 across all cue patterns, which no systematic decision strategy would predict. These participants were therefore classified as using a random choice strategy (RAND). 


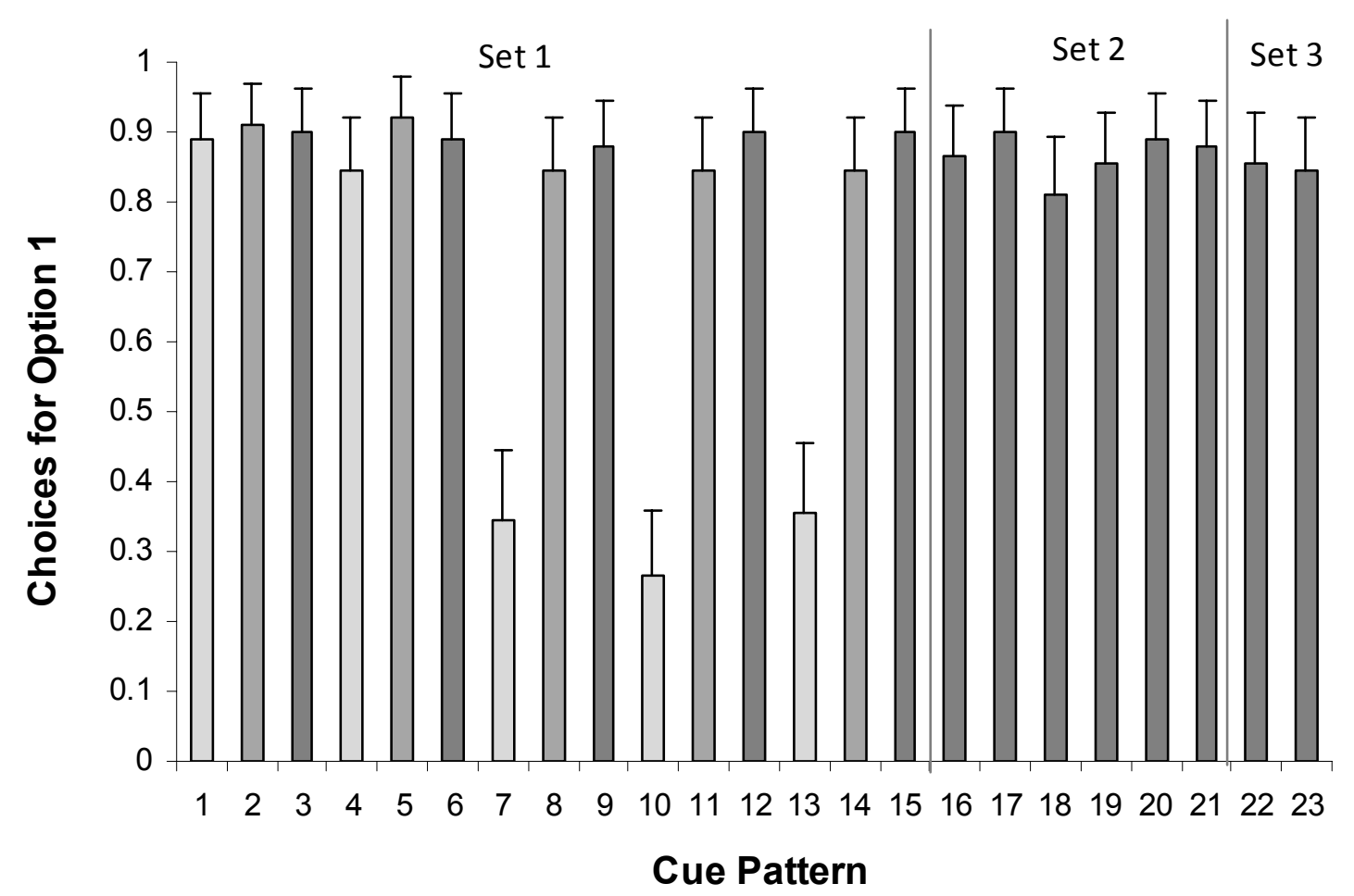

Figure 1. Percentage of choices of option 1 in Experiment 1. The "Positive 01" manipulation within set 1 is indicated by different grayscales. Error bars indicate 95 percent confidence intervals.

Based on this analysis, it can be concluded that at least 11 participants did not concentrate on the most valid cue only, but instead used information from all three cues, which might be explained by EQW or WADD strategies. However, an EQW strategy further predicts that in cue patterns $4,8,11$, and 18, an equal distribution of choices of options 1 and the other options should be observed, whereas WADD predicts choices of option 1 only. For the 11 participants yet to be classified, the distribution of choices in these cue patterns was examined using $\chi^{2}$ tests. Specifically, observed choices were tested against an equal distribution of choices of option 1, compared with the sum of choices of the other options (i.e., 50:50 choices of option 1 vs. choices of options $2+3$ ). There was a precisely equal distribution for one person, who was accordingly classified as an EQW user. For the remaining 10 participants, choices significantly deviated from an equal distribution; these participants clearly preferred option 1 ( $p$ $<.05)$. Having apparently used information from all three cues and also taking into account the cue validities, they were classified as WADD users. The results of the strategy classification are summarized in the top row of Table 3. It can be seen that about two thirds of the participants used a WADD strategy, whereas only a few individuals used EQW or LEX/TTB strategies and ignored information. Thus, in contrast to earlier findings, simple LEX/TTB strategies were not predominantly used under time pressure. 


\begin{tabular}{|c|c|c|c|c|}
\hline & \multicolumn{4}{|c|}{ Decision Strategies } \\
\hline & LEX/TTB & EQW & WADD & RAND \\
\hline & & & t 1 & \\
\hline \multirow[t]{2}{*}{ Time Pressure } & $2(13 \%)$ & $1(7 \%)$ & $10(67 \%)$ & $2(13 \%)$ \\
\hline & \multicolumn{4}{|c|}{ Experiment 2} \\
\hline Lenient Time Limit & $4(27 \%)$ & $1(7 \%)$ & $10(67 \%)$ & 0 \\
\hline Medium Time Limit & $5(33 \%)$ & $3(20 \%)$ & $7(46 \%)$ & 0 \\
\hline \multirow[t]{2}{*}{ Severe Time Limit } & $14(93 \%)$ & 0 & $1(7 \%)$ & 0 \\
\hline & \multicolumn{4}{|c|}{ Experiment 3} \\
\hline \multicolumn{5}{|c|}{ Time Pressure in Complex } \\
\hline Decision Tasks & $13(21 \%)$ & 0 & $50(79 \%)$ & 0 \\
\hline
\end{tabular}

Strategy Classification Based on Decision Times. The median decision time was found to be very low: Half of the decisions were made in less than 1.1 seconds $(M D=1.07 \mathrm{~s}, M=1.53 \mathrm{~s}$, $S D=1.51 \mathrm{~s}$, skew $=6.19$, kurtosis $=65.11)$. Thus, it can be concluded that participants actually followed the time limit instructions. The short decision time makes it fairly unlikely that individuals were able to deliberately integrate cue values and cue validities (WADD $\left.{ }_{\text {del }}\right)$. To strengthen this argument, we carried out a study in which participants were instructed to deliberately apply a $W{ } D_{\text {del }}$ strategy to similar decision tasks with two options and three cues (Glöckner, 2006). The observed average decision time was 20.5 seconds $(S E=2.2 \mathrm{~s}$ ), which lies far above the decision times observed in this experiment. Lohse and Johnson (1996) report comparable decision time predictions for the application of $\mathrm{WADD}_{\mathrm{del}}$ in an open Mouselab. For decisions with two options but two or seven attributes, the partially empirically derived decision time predictions were 7.7 and 29.1 seconds.

Decision time data were log-transformed to the basis of 10 to reduce the influence of outliers and the skewness and kurtosis of the distribution (Glass \& Hopkins, 1996). The transformed data points were fairly normally distributed $(M D=3.03, M=3.09, S D=0.26$, skew $=1.05$, kurtosis $=1.27)$. To analyze decision times, a $23($ CUE PATTERN) x $6($ ORDER) repeated measurement ANOVA was conducted, with log-transformed decision time as the dependent variable. CUE PATTERN and ORDER were used as within-subject factors. Each cue pattern was presented in six different versions, which were presented in a random order. Thus, the factor ORDER ranged from first (1) to last (6) repetition of the cue pattern (and replaced the factor VERSION in the analysis). A Greenhouse-Geisser correction was used because Mauchly's test turned out to be significant, indicating that the assumption of sphericity was violated. The same correction was also used in all following analyses, where indicated. The main effect for CUE PATTERN was highly significant, $F(4.9,68.2)=24.04, p<.001, \eta^{2}=$ 
$.63{ }^{2}$ Thus, it can be concluded that decision times differ systematically between cue patterns (Figure 2), which speaks against the sole application of a $\mathrm{WADD}_{\text {del }}$ strategy.

As expected, there was also a significant main effect for the factor ORDER, $F(1.6,22.8)=$ $7.47, p=.006, \eta^{2}=.35$. Although the time needed for the decision was already very low in the first presentation of each cue pattern, decision times further decreased in later repetitions, indicating learning effects. For this factor, the mean values for log-transformed decision times, starting with the first presentation (with $S E$ in parentheses), were 3.17 (0.037), 3.08 (0.039), 3.08 (0.039), 3.07 (0.045), 3.05 (0.042), and $3.06(0.041)$.

The effect of the factor CUE on decision times was analyzed using a repeated-measurement ANOVA with CUE as a within-participants factor. The main effect for CUE turned out to be significant, $F(2,28)=4.45, p=.021, \eta^{2}=.24$. However, in contrast to the predictions derived from a LEX/TTB strategy, decision times significantly decreased with the increasing number of cues needed to differentiate according to a LEX/TTB strategy $\left(M_{1}=3.097, S E=0.006 ; M_{2}\right.$ $\left.=3.070, S E=0.009 ; M_{3}=3.056, S E=0.016\right)$. Thus, decision time data converge with choice data in indicating that participants did not predominantly use a LEX/TTB strategy.

\section{Figure 2}

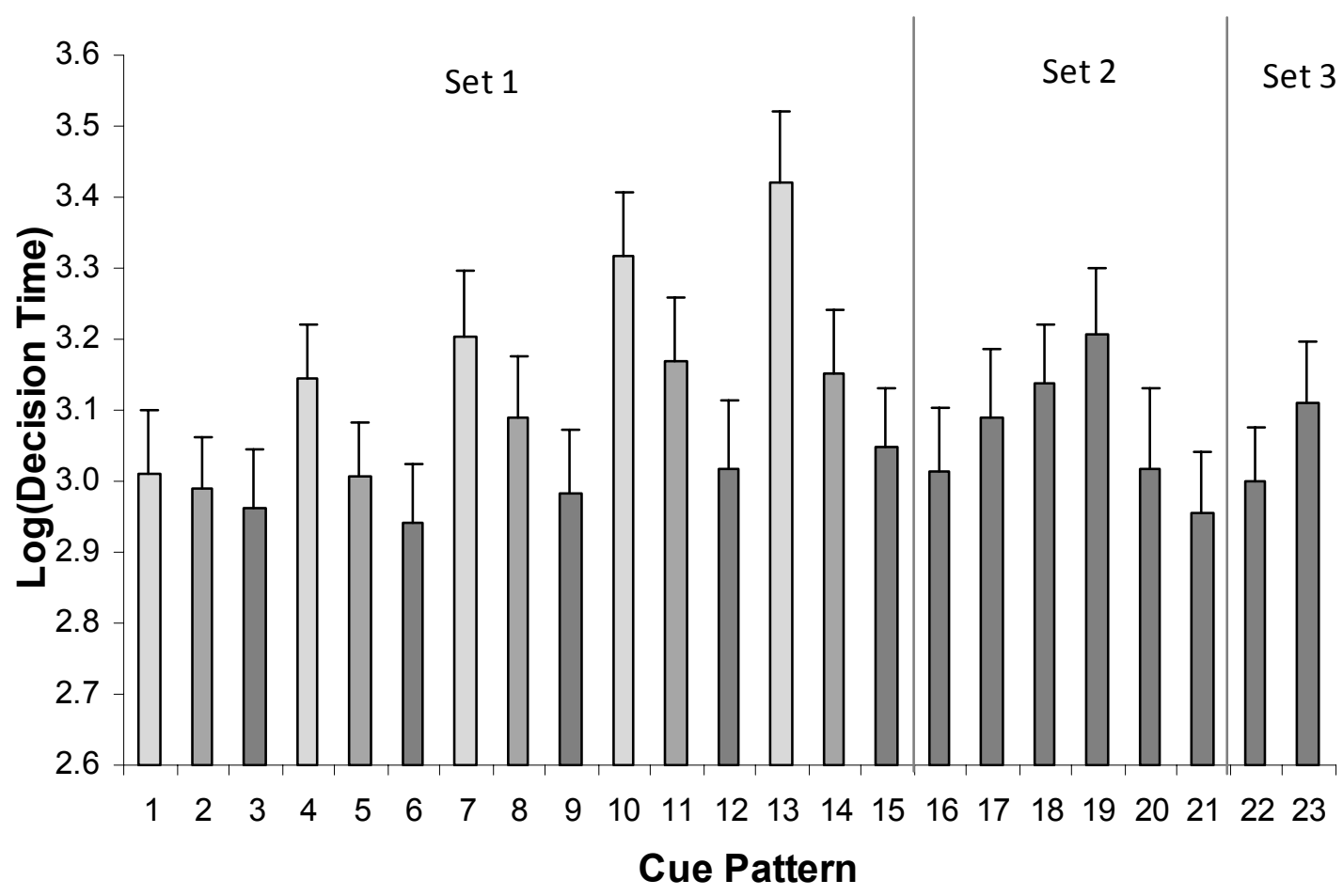

Figure 2. Log-transformed decision times in Experiment 1. The "Positive 01" manipulation within set 1 is indicated by different grayscales. Error bars indicate 95 percent confidence intervals.

2 The effect-size measures reported in this paper are all partial $\eta 2$-values. Therefore, values do not add up to 1. 
The effects of the factors PRO_OPTION1 and PRO_OPTION2/3 were investigated using a 3 (PRO_OPTION1) X 5 (PRO_OPTION2/3) repeated measurement ANOVA with logtransformed decision times as the dependent variable. The analysis was run for set 1 only because there was no systematic manipulation of the factors in the remaining sets (cf. Table 2). There were highly significant main effects for PRO_OPTION1, $F(1.3,17.9)=31.8, p<.001$, $\eta^{2}=.69$, and PRO_OPTION2/3, $F(2.9,41.1)=15.0, p<.001, \eta^{2}=.52$, and a significant interaction between both factors, $F(2.2,30.4)=36.9, p<.001, \eta^{2}=.73$. As predicted by $\mathrm{WADD}_{\text {auto }}$ (but contrary to the predictions of $\mathrm{WADD}_{\text {del }}$, LEX/TTB, and EQW), decision times decreased with an increasing number of cues favoring option 1 and increased with an increasing number of cue values favoring options 2 and 3 (Figure 2). Decision times were particularly high for the critical cue patterns $(7,10,13)$, accounting for the interaction effect.

\section{Discussion}

Our results indicate that the majority of the participants $(67 \%)$ considered information on all three cues as well as cue validities. Their choices were in line with a WADD strategy. Most importantly, individuals were capable of successfully applying this strategy within less than 1.5 seconds on average $(M D=1.07 \mathrm{~s})$. Although set under time pressure, participants refrained from using simple deliberate strategies like LEX/TTB or EQW. Consequently, the major findings from Mouselab studies could not be replicated when individuals had unconstrained access to relevant information. Our results suggest that individuals can capitalize on remarkable abilities for complex information integration and multiple-reason decision making. Considering the decision times observed in the above-mentioned study, in which participants were instructed to deliberately calculate weighted sums (Glöckner, 2006), it is unlikely that individuals deliberately computed a $\mathrm{WADD}_{\text {del }}$ strategy, because the median decision time of less than 1.1 seconds is far below the time necessary for calculating $\mathrm{WADD}_{\text {del }}$ or similar weighted additive strategies.

We argue that these results emphasize the importance of automatic processes in decision making. As proposed by different authors (e.g., Hammond et al., 1987; Kahneman \& Frederick, 2002), individuals seem to possess the ability not only to use simple deliberate heuristics but also to apply decision strategies based on automatic processes that lead to choices according to a weighted additive information integration (i.e., $\mathrm{WADD}_{\text {auto }}$ ). Even under time pressure, these automatic strategies appear to have guided the decisions of the majority of participants in our studies. By disentangling the limitations of information search and information integration, we were able to demonstrate that the cognitive capacity for information integration is not as limited as generally assumed by proponents of the bounded rationality approach (e.g., Gigerenzer, 2004).

The results of Experiment 1 conflict with the well-established findings from Mouselab studies showing that when there is severe time pressure, simple LEX/TTB strategies are usually employed (e.g., Payne et al., 1988). As argued above, Mouselab fosters the application of 
LEX/TTB strategies, because information search consumes considerable resources (e.g., time required to perform the appropriate mouse movements). We remedied this problem by using an open information display and a choice-based strategy classification method. ${ }^{3}$ Our findings indicate that limitations of their information integration capacity is not always the major reason for individuals adopting simple strategies when subjected to time pressure. There is instead a straightforward alternative interpretation: Lacking the time to look up all information, individuals concentrate on the most important pieces. Our results converge with several recent findings showing that people tend to use WADD strategies instead of simple heuristics if information can be directly accessed in the environment (Bröder, 2000a; 2003; Glöckner, 2006).

Results on decision times further strengthen our point and provide converging evidence with the choice analysis. The decision times clearly speak against the application of a deliberative compensatory strategy $\left(\mathrm{WADD}_{\text {del }}\right)$ and instead support the assumption that weighted additive procedures are performed by the automatic system (WADD ${ }_{\text {auto }}$ ). It might be argued that individuals could have used the following heuristic: "Choose the option favored by cue 1 unless consensually outvoted by cues 2 and 3."4 Obviously, this is also a multiple-reason strategy because it takes into account all cue values and at least ordinal information about cue validities; nevertheless, it would be easier to apply than a $\mathrm{WADD}_{\text {del }}$ strategy. However, the observed systematic variations of decisions times clearly rule out its application. Specifically, this heuristic could not explain the significant main effects for the number of positive cue values for option 1 (pro_option1) and the number of positive cue values for options 2 and 3 (pro_option $2 / 3$ ). Overall, it seems unlikely that simple heuristics can account for the differentiated findings concerning decision times.

\section{Experiment 2}

In Mouselab studies, it is a well documented finding that individuals switch to simple, noncompensatory strategies if task constraints (e.g., time) become severe (e.g., Rieskamp \& Hoffrage, 1999). We argued that this finding might only apply to situations in which individuals use deliberate decision strategies and Mouselab might induce the application of such strategies. In Mouselab studies, time pressure manipulations, for instance, might simply constrain the depth of information search, because the motor activity needed to move the mouse to the appropriate boxes is time consuming. The first experiment provided compelling evidence in favor of this claim. As a caveat, however, we used a decision problem that has never been

From a methodological perspective, it needs to be kept in mind that choice-based strategy classification entails the problem that WADD predictions dovetail with predictions from LEX/TTB and EQW strategies (Bergert \& Nosofsky, 2007; Bröder, 2000b; Glöckner, 2006; Glöckner, in press). This is because the latter are submodels of the former (e.g., persons who have equal cue validities for all cues but use a WADD strategy are misclassified as EQW users that ignore cue weights per se). Thus, the proportions of WADD users reported in this and the following studies have to be considered a conservative estimate of the real proportions, and it is highly possible that more participants used a WADD strategy. Note, however, that this only strengthens our argument.

We thank Peter Ayton for suggesting this alternative heuristic. 
employed in Mouselab studies before. To rule out that results are specific to our problem domain, we ran a second experiment that used the same types of problems but employed the classic Mouselab tool. If the above reasoning is correct, we should be able to replicate those findings documented in the literature. Specifically, in a Mouselab with hidden information, participants should switch to simple, noncompensatory strategies if time limits become severe.

We manipulated time limits on three levels. A lenient time limit condition was designed to allow for the repeated inspection of all the information. A medium time limit condition provided just enough time to look up each piece of information once. A severe time limit condition did not allow all pieces of information to be looked up, but it provided approximately the self-selected average decision time that individuals used in Experiment 1 . We expected that the majority of individuals would apply a WADD strategy under the lenient and medium time limit conditions but adopt a LEX/TTB strategy under the severe time limit condition.

In the classic Mouselab program, information search parameters can be recorded. We considered two important parameters of information search: the number of information boxes opened per cue and the direction of search. We use the PATTERN index to measure direction of search (also called SI-index; Payne et al., 1988; for a critical view, see Böckenholt \& Hynan, 1994; see also Footnote 6 below). PATTERN indexes the relative proportion of cuebased and option-based transitions between information boxes. Given the acquisition of a particular piece of information, a cue-based transition means that the next acquisition involves the same cue but a different option; an option-based transition means that the next acquisition involves the same option but a different cue. PATTERN is calculated by subtracting the number of cue-based transitions from the number of option-based transitions and dividing this difference by the sum of both transitions. Thus, PATTERN indicates whether individuals' search for information is more cue-based or option-based. It ranges from -1 to 1 . Negative values indicate more cue-based and positive values more option-based search. Hence, negative scores are usually interpreted as evidence for noncompensatory decision strategies like LEX/TTB, whereas positive scores are taken as evidence for compensatory decision strategies like WADD (although it has to be acknowledged that the score only measures the direction of information search, not information integration itself).

\section{Method}

Participants and Design. Fifteen students (9 female, 6 male) from the University of Heidelberg participated in a 30-minute study and were compensated for participation either by course credit or a flat fee of 4 euros. Again, decision tasks were manipulated within participants by using 23 different cue patterns in six different versions (see Table 2). Time limit was manipulated on three levels as a further within-participants factor (lenient: $8 \mathrm{~s}$, medium: $3 \mathrm{~s}$, severe: $1.5 \mathrm{~s}$ ). The six versions of each cue pattern were equally assigned to one of the time limit conditions using a random procedure. Thus, the factor VERSION was nested within the 
factor TIME LIMIT, resulting in a 23 (CUE PATTERN) x 3 (TIME LIMIT) x 6 (VERSION) nested within-participants design.

Materials and Procedure. We used the same cover story, instructions, and materials as in Experiment 1. Again, cue information was presented in an information matrix with options displayed in columns and cues in rows (Figure 3). In contrast to the previous study, information was hidden in boxes. Each information box opened automatically when hit by the mouse cursor. First, participants were introduced to the task and informed about the cue validities. After becoming familiarized with Mouselab, they completed eight test trials. The following 138 target decision tasks were presented in three blocks, each with a different time limit. Time limits increased over the three blocks of trials. Blocks were separated by 1-minute breaks (black screen). For each decision task, participants could move the mouse to access information. A piece of information was visible only as long as the cursor was held on the information box. A time bar was shown at the top of the screen to inform participants of time limits. The length of the bar decreased in proportion to the elapsed time. Immediately after the decision task was presented, the bar began counting down time.

Figure 3

Search for information and select a vendor by clicking on it.

\begin{tabular}{|l|l|l|l|}
\hline \multicolumn{2}{|l|}{} & \multicolumn{2}{|l|}{ Option 3 } \\
\hline & Option 1 & Option 2 & \\
\hline Tester 1 & & $\mathbf{r}$ & \\
\hline Tester 2 & & & \\
& & & \\
\hline Tester 3 & & & \\
\hline
\end{tabular}

Figure 3. Mouselab presentation used in Experiment 2.

Again, participants were asked to make accurate decisions and to proceed as quickly as possible. Furthermore, they had to make their choices within the time limit; after the allotted time had elapsed, no further information search was possible. Nevertheless, participants were forced to make a decision and were subsequently reminded to keep within time limits. Choices, decision times, and information-search parameters (opened information boxes and 
time for opening) were recorded. ${ }^{5}$ Decision times were measured from the onset of the decision task to the selection of the option. Options were selected by mouse click; the mouse cursor was initially positioned in the upper left-hand corner of the screen. Each decision task was started by clicking a button on an introductory screen. The Mouselab software was programmed in Visual Basic 6.0 and was run on IBM-compatible computers.

\section{Results}

Strategy Classification Based on Choices. The same method as in the previous experiment was used to classify strategies. Each time limit block was analyzed separately. Note that the reduced number of analyzed choices reduced the statistical power and thereby increased the probability of LEX/TTB and EQW classifications. As explained in the results section of Experiment 1 , two individual $\chi^{2}$-tests had to turn out significant for one participant to be classified as WADD user. With only one third of the number of observations, it becomes less likely that existing effects will be detected at a specified alpha level (i.e., the beta error increases). Accordingly, the likelihood that a WADD user is mistakenly classified as an EQW or LEX/TTB user increases substantially. In compensation, we used an increased alpha level of $\alpha=.10$ in both tests. First, we analyzed whether the proportion of choices of option 1 (vs. choices of options $2+3$ ) in cue patterns 7,10 , and 13 differed from the proportion of choices of option 1 in the remaining cue patterns, using individual $\chi^{2}$-tests of independence. Second, we tested the observed proportions across options (i.e., option 1 vs. options $2+3$ ) in patterns $4,8,11$, and 18 against an equal distribution. It turned out that under the lenient time limit condition, the majority of participants used a WADD strategy (Table 3). Under the medium condition, participants mainly retained their decision strategy. Three former WADD users switched to the EQW and LEX/TTB strategies. When the time limits were severe, almost all the participants turned to a LEX/TTB strategy. Thus, choice data are in line with our expectation that decision strategies only change if time pressure prevents all pieces of information from being inspected (i.e., under severe time limits).

Manipulation Check for the Factor TIME LIMIT. A 23 (CUE PATTERN) x 3 (TIME LIMIT) x 2 (REPEAT) repeated measurement ANOVA, with log-transformed decision times as dependent variables, revealed a significant effect for TIME LIMIT, $F(1.3,18.4)=457.4, p<$ $.001, \eta^{2}=.97$. This indicates that our time limit manipulation was successful. With decreasing time limits, actual decision times decreased. The median decision times for the lenient, medium, and severe time limit conditions in seconds were 4.28, 2.56, and 1.49 respectively. Furthermore, CUE PATTERN was found to have a significant main effect, $F(3.1,44.0)=5.80, p$ $<.001, \eta^{2}=.29$. For the critical patterns 7,10 , and 13, the decision times were again particularly high. Similarly high decision times were found for cue patterns 4, 17, 18, 19, and 23. A

5 For pragmatic reasons, information-search data were recorded only for the first 20 inspected information boxes per decision task. 
considerable portion of decisions ( $22 \%$ vs. $49 \%)$ under the medium and severe time limit conditions was made after the time limit allotted for the information search had elapsed.

Strategy Classification Based on Information Search. The distribution of inspected information boxes per cue was analyzed using a 3 (CUE) x 3 (TIME LIMIT) repeated measurement ANOVA, with the numbers of viewed information boxes as a dependent variable. The main effect for CUE was highly significant, $F(1.9,26.0)=45.52, p<.001, \eta^{2}=.76$, indicating that participants looked up more pieces of information for more valid cues (Table 4). There was also a significant main effect for TIME LIMIT, $F(1.4,19.3)=92.6, p<.001, \eta^{2}=.87$. The number of opened information boxes also decreased parallel to decreases in the time limit. As indicated by the information box index in Table 4 (which is calculated by dividing the number of opened information boxes by the number of available ones), under lenient time limits, participants inspected each information box more than once; under medium time limits, each information box was opened once on average; under severe time limits, only 61 percent of the information boxes were inspected. The interaction between the factors CUE and TIME LIMIT was also significant, $F(2.1,29.8)=18.7, p<.001, \eta^{2}=.57$. It was found that participants focused their information search on the most valid cue even more when time limits decreased.

\section{Table 4}

Information-Search Parameters Experiment 2

\begin{tabular}{|c|c|c|c|c|c|c|}
\hline & \multicolumn{6}{|c|}{ Information-Search Parameters } \\
\hline & \multicolumn{4}{|c|}{ Information Box Index } & \multicolumn{2}{|c|}{ PATTERN } \\
\hline & Cue 1 & Cue 2 & Cue 3 & $M$ & $M$ & $S E$ \\
\hline Lenient Time Limit & 1.51 & 1.49 & 1.01 & 1.34 & .05 & .10 \\
\hline Medium Time Limit & 1.31 & 1.16 & 0.60 & 1.02 & .08 & .11 \\
\hline Severe Time Limit & 1.16 & .49 & 0.16 & 0.61 & -.42 & .09 \\
\hline
\end{tabular}

Note. The information box index is a measure of the number of information boxes opened divided by the number of boxes available. A value of 1 indicates that the number of opened information boxes was equal to the number of available information boxes; lower values indicate that fewer boxes were opened than available. Higher values indicate that boxes have been opened repeatedly. The PATTERN index indicates whether information searches were predominantly cue-based (negative values) or option-based (positive values).

To further investigate search strategies, the information search index PATTERN was computed (Payne et al., 1988). ${ }^{6}$ We conducted a repeated measurement ANOVA with the differ-

6 Note that transitions in which the acquisition involves both another cue and another option are not considered in the index and that the PATTERN index for each participant was computed using the total number of cue-based and option-based transitions for the 46 decision trials in each time limit condition. It has been argued that the former could lead to biased strategy classification results if the number of options and the number of cues (or dimensions) in the Mouselab matrix differ (Böckenholt \& Hynan, 1994). This was not the case in our experiment. Nevertheless, we additionally calculated the unstandarized $S M^{*}$ index that also takes into account all other transitions (Böckenholt \& Hynan, 1994). As expected, this did not change our results substantially $\left(S M *\right.$ lenient $T P=.06, S M^{*}$ medium $T P=.07, S M^{*}$ sever $\left.T P=-.30\right)$. 
ent PATTERN scores for the time limit conditions as dependent variables. There was a significant effect for TIME LIMIT, $F(1.3,18.6)=14.0, p=.001, \eta^{2}=.50$. Under the first two conditions, there was a slight preference for option-based searches. Under the severe time limits, in contrast, individuals showed a strong preference for cue-based searches (Table 4; right column). Two contrasts were computed to further pinpoint this effect. It turned out that there was no difference in the PATTERN scores between the lenient and the medium time limit conditions, $F(1,14)=.16, p=.70, \eta^{2}=.01$. However, the difference between the severe time limit condition and medium/lenient time limit conditions was highly significant, $F(1,14)=$ $16.32, p<.01, \eta^{2}=.54$. Thus, as expected, patterns of information search changed only when decision time was too short to investigate all pieces of information.

For each person and each time limit block, we further analyzed whether results on the PATTERN index dovetail with those of strategy classification based on choices. In $73 \%$ percent of the 45 comparisons (each comparison was computed for three time limit conditions per person), results of both measures converged.

\section{Discussion}

The second experiment examined whether changes in decision strategies in Mouselab experiments under time pressure (e.g. Payne et al., 1988) are due to limitations in information search instead of limitations in cognitive capacity, as is generally assumed. It was found that when sufficient time to inspect all of the information was allotted, choices were in line with a WADD strategy. Under the severe time limit of 1.5 seconds, it was no longer possible to inspect all the information boxes; participants then used a LEX/TTB strategy, inspecting only the information on the most valid cue. Together, the results of Experiment 1 and 2 provide evidence that at least in some experimental settings the use of simple, noncompensatory strategies (e.g., LEX/TTB) is indeed caused by constraints of information search rather than by limitations of cognitive capacity.

In the two experiments, choices and decision time patterns were shown to be in line with our hypothesis that individuals are able to quickly integrate multiple pieces of evidence in a weighted additive manner. There is conclusive evidence that the majority of individuals takes available pieces of information into account and considers them according to their validity. Thus, our results are difficult to explain with fast and frugal heuristics (Gigerenzer et al., 1999) that ignore either cue values or cue validities. The applied decision strategies appear not to be based on one or a few reasons but instead capitalize on the wealth of the information given.

Nevertheless, the findings could still be challenged in two ways. First, it might be questioned whether the results generalize to more complex decision tasks. One possible hypothesis is that individuals encode the array of information as a constellation. This constellation could be compared with different prototypes or exemplars (Juslin, Olsson, \& Olsson, 2003; Olsson, 
Enkvist, \& Juslin, 2006), or mere perceptual pattern recognition processes could be used to reach a decision quickly (e.g., by identifying a specific series of + and -). This might be particularly the case in simple tasks in which cues are always presented in the same order. Second, one might speculate whether the results evidence application of "complex heuristics" such as TTB-CONFIGURAL (Garcia-Retamero, Wallin, \& Dieckmann, 2007): Participants could have used fast and frugal heuristics that were not based on single cues but rather on complex ones, such as configurations of cue values (which are then applied in the order of their validity). Individuals could have learned to react appropriately to certain cue patterns by identifying the complete constellations of the three cues (e.g., the most important cue points towards A, all others point towards B) or parts of them (but see discussion of Experiment 1). Findings by Nosofsky and Bergert (2007) lend initial support for the application of TTBCONFIGURAL in multiple cue decision tasks. To critically test these alternative interpretations, we conducted a third experiment.

\section{Experiment 3}

In this study, we kept the constellation of cue information constant and manipulated the validity of only one cue without changing the constellation (i.e., without changing the order of cues in the cue hierarchy). If decisions are based solely on the conceptual constellation of cue information, this manipulation should not influence decisions. Furthermore, the presentation order of cues in the information matrix was randomized to prevent mere perceptual pattern recognition processes. More complex decision problems with six cues and two options were used. Confidence judgments were measured as an additional dependent variable. According to a LEX/TTB strategy, confidence judgments should depend on the validity of the differentiating cue only. According to a $\mathrm{WADD}_{\text {auto }}$ strategy, confidence judgments should depend on the difference between the weighted sums of cue values and cue validities for the available options.

\section{Method}

Participants and Design. Sixty-three students (55 female, 8 male) from the University of Erfurt participated in a 20-minute-long experiment, which was part of a one-hour experimental battery of thematically unrelated studies. Students received 6 Euros for their participation in the entire hour. Decision tasks were again manipulated within participants, resulting in a 6 (CUE PATTERN) x 4 (CUE VALIDITY) x 3 (REPETITION) design. 


\begin{tabular}{|c|c|c|c|c|c|c|c|c|c|c|c|c|}
\hline & \multicolumn{12}{|c|}{ Cue Pattern } \\
\hline & \multicolumn{2}{|c|}{1} & \multicolumn{2}{|c|}{2} & \multicolumn{2}{|c|}{3} & \multicolumn{2}{|c|}{4} & \multicolumn{2}{|c|}{5} & \multicolumn{2}{|c|}{6} \\
\hline & $A$ & $\mathrm{~B}$ & A & B & A & B & $A$ & $\mathrm{~B}$ & $A$ & B & $A$ & B \\
\hline Cue $1(p=.80$ to .95$)$ & + & - & + & - & + & - & + & - & + & - & + & - \\
\hline Cue $2(p=.75)$ & - & + & - & - & - & + & - & - & - & + & + & - \\
\hline Cue $3(p=.70)$ & - & - & - & - & - & + & - & - & - & + & - & - \\
\hline Cue $4(p=.65)$ & - & - & - & - & - & - & - & - & - & + & - & - \\
\hline Cue $5(p=.60)$ & - & - & - & - & - & - & - & + & - & + & - & + \\
\hline Cue $6(p=.55)$ & - & - & - & + & - & - & - & + & - & + & - & + \\
\hline
\end{tabular}

Note. The six cue patterns used in Experiment 3 are depicted in a matrix format. Cues are shown in the left-hand column. The percentage of correct predictions $p$ of each cue is given in parentheses. A and B represent options.

Materials and procedure. Six cue patterns were used (Table 5). In cue patterns 1, 2, and 6, an equal number of cues had positive cue values for both options. In cue patterns 3,4 , and 5 , the most valid cue made a prediction contrary to at least two other cues. Cue patterns were repeated three times with randomized orders of options and cues (REPETITION). Again, we provided individuals with explicit information about the probability of correct predictions made by each cue. This probability was varied for the most valid cue from .80 to .95 in steps of .05 (CUE VALIDITY). The probabilities were constant for the remaining cues 2 to 6 at levels of $.75, .70, .65, .60$, and .55 .

Independent of the cue validity manipulation, the LEX/TTB strategy predicts choices of option $\mathrm{A}$ in all six cue patterns, because the most valid cue always points towards this option. The EQW strategy predicts choices of options B in cue patterns 3, 4, and 5 and a random selection of options in the remaining ones. Independent of the manipulation of the validity of cue 1, the WADD strategy predicts choices of option A in cue patterns 1,2, and 6.

Choices in WADD strategies crucially depend on individuals' transformation of given accuracy probabilities of cues into cue weights. The simplest possibility is to follow an ignorant $W A D D$ strategy and to use probabilities as cue weights without any transformation (e.g., .55 for cue 6). In cue patterns 3,4 , and 5 such an ignorant WADD strategy would make predictions for option B only. Note that such a strategy could easily be misleading because it does not take into account that cues with a probability of .50 are uninformative and should be ignored (cf. Experiment 1). It would be more appropriate for participants to transform provided probabilities into cue weights so that information about cues with a probability of .50 is weighted 0. Individuals who use an ignorant WADD strategy would select option B in cue patterns 3,4 , and 5 , independent of the cue validity manipulation. Individuals who take into 
account the problem and correct their decision weights should show decreasing choices of option B with increasing validity of cue 1.

The cue validity manipulation should only influence choices if participants use decision strategies that are based on processes that integrate information in a weighted additive manner; no influence of the manipulation on choices would be predicted if individuals base their decision simply on the constellation of cues. Thus, the manipulation of cue validities was used for testing the (second) alternative explanation raised above.

Participants made their choices via mouse click on the option (Figure 4). After each choice, individuals were asked to indicate their confidence in their decision on a continuous horizontal scale: "Please indicate how certain you are of your decision!" The endpoints of the scale were labeled "very uncertain" and "very certain." The scrollbar was presented below the information matrix, which remained visible until the judgment was made. After each decision, an instruction screen was shown. Clicking a "continue" button ensured that the cursor was always positioned in the middle of the screen when the individual started to work on the next decision task.

Figure 4

\begin{tabular}{|l|c|c|}
\hline & $\begin{array}{c}\text { Oranges A } \\
\text { Choose }\end{array}$ & $\begin{array}{c}\text { Oranges B } \\
\text { Choose }\end{array}$ \\
\hline $\begin{array}{l}\text { Tester } 1 \\
(90 \% \text { correct })\end{array}$ & $\mathbf{-}$ & - \\
\hline $\begin{array}{l}\text { Tester } 2 \\
(60 \% \text { correct })\end{array}$ & - & - \\
\hline $\begin{array}{l}\text { Tester } 3 \\
(70 \% \text { correct })\end{array}$ & - & $\mathbf{+}$ \\
\hline $\begin{array}{l}\text { Tester } 4 \\
(75 \% \text { correct })\end{array}$ & - & - \\
\hline $\begin{array}{l}\text { Tester } 5 \\
(65 \% \text { correct })\end{array}$ & - & - \\
\hline $\begin{array}{l}\text { Tester } 6 \\
(55 \% \text { correct })\end{array}$ & - & $\mathbf{-}$ \\
\hline
\end{tabular}

Figure 4. Presentation format of decision tasks used in Experiment 3.

After a learning trial, individuals were presented with 72 decision tasks comprising different versions of the six cue patterns presented in Table 5. Each CUE PATTERN was realized for all four CUE VALIDITY conditions and each of the resulting decision tasks was repeated three times. Decision tasks were presented in individually randomized order. The order of the options and the order of the cues in each decision were also individually randomized for each trial. 


\section{Results}

Strategy Classification Based on Aggregate Choices. Proportions of choices of option B in the six CUE PATTERNS and the four CUE VALIDITY conditions are depicted in Figure 5. There were very few decisions for option $\mathrm{B}$ in cue patterns 1,2 , and 6 , but a considerable proportion of choices of option B in cue patterns 3,4 , and 5, with choice proportions for option $B$ decreasing with increasing validity of the first cue. To test whether choices differ significantly between the six cue patterns, a $\chi^{2}$ - test against an equal distribution of choices of option $\mathrm{B}$ across the six cue patterns was conducted. This analysis produced a highly significant effect, $\chi$ $(5 ; N=789)=1176.6, p<.001$. Thus, contrary to the predictions of a LEX/TTB strategy, aggregated choices differ significantly between cue patterns, indicating that individuals based their decisions on multiple pieces of information rather than on the most valid cue. To test whether choices differ between levels of CUE VALIDITY for cue 1 , a $\chi^{2}$ - test against an equal distribution of choices of option B in the four CUE VALIDITY conditions was conducted. The test turned out to be highly significant, $\chi(3 ; N=789)=86.5, p<.001$. Thus, against the predictions of the EQW strategy, choices were influenced by the cue validity manipulation, indicating that cue information was considered according to its validity. The significant effects of the CUE VALIDITY manipulation rules out the hypothesis that individuals simply react to constellations of cues because the constellation was held constant and only validities of the most valid cue varied.

Figure 5

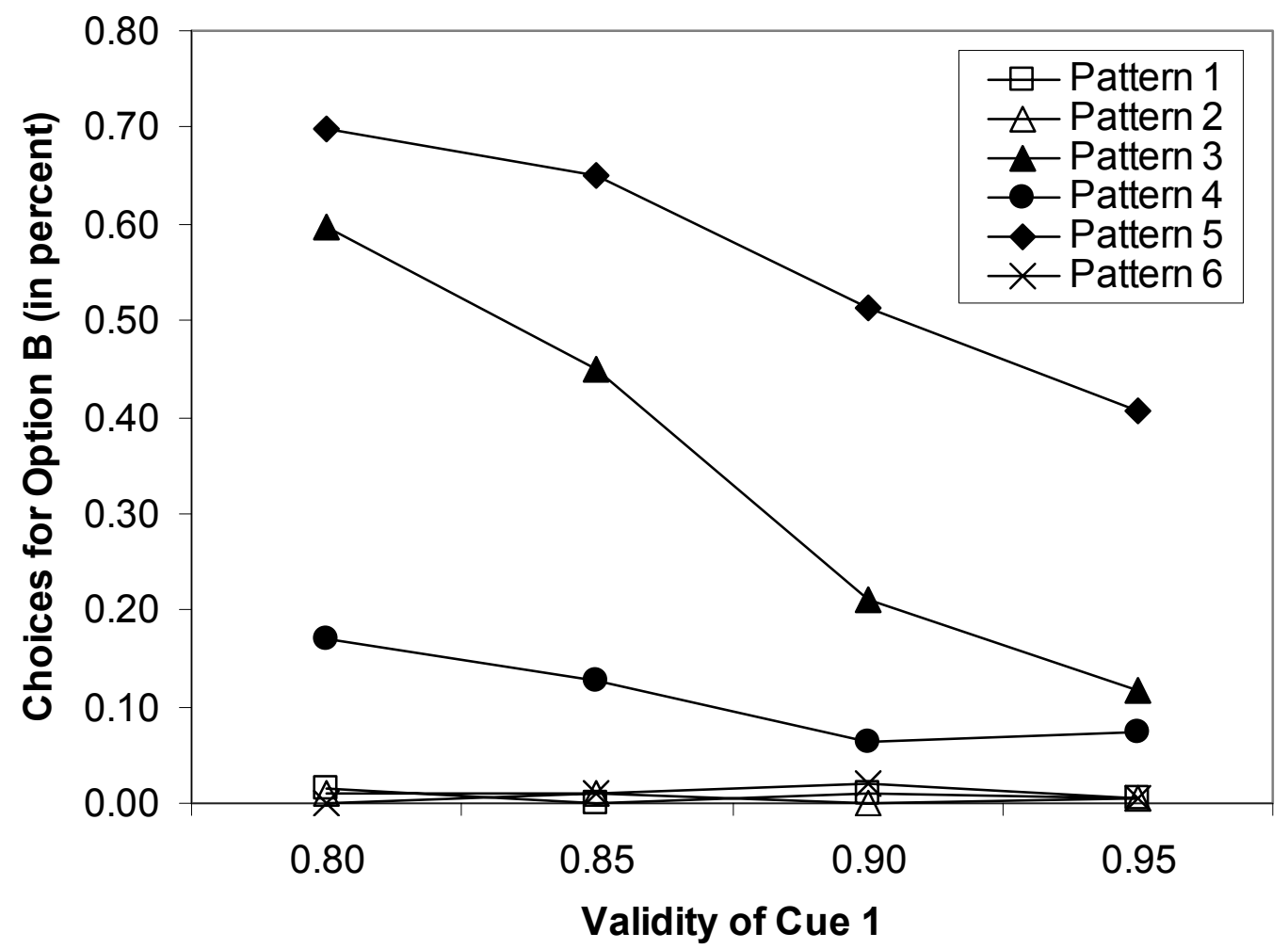

Figure 5. Percentage of choices of option 2 in Experiment 3. 
Strategy Classification Based on Individual Choices. To investigate decision strategies more closely, individual choice patterns were analyzed in the same way as in the previous experiments. For each individual, two $\chi^{2}$ - tests were conducted to test against the predictions of the LEX/TTB and the EQW strategies. First we tested whether the proportion of choices of option A or B were the same in the cue patterns 1,2, and 6 as compared to the cue patterns 3, 4, and 5 using a $\chi^{2}$ - test of independence. A significant difference would indicate that individuals did not use a LEX/TTB strategy. Second we tested whether the choices of option A and B were equally distributed in cue patterns 1,2 , and 6 . A significant deviation from the equal distribution would indicate that individuals did not use an EQW strategy but instead acknowledged cue validities. A significance level of $\alpha=.05$ was applied in both clusters of tests. If one of the tests failed to reach this level of significance, an individual was classified as LEX/TTB or EQW user, respectively. The results of the strategy classification are shown in Table 3 (last row). In line with the results on the aggregate level, the majority of individuals decided in accordance with a WADD strategy. Only a minority of individuals seemed to use a LEX/TTB strategy.

\section{A 6 (CUE PATTERN) x 4 (CUE VALIDITY) x 3 (REPETITION) x 2 (DECISION TIME} VS. CONFIDENCE) MANOVA was conducted to investigate the simultaneous effect of the within-participants manipulations on decision times and confidence judgments. The relevant factors CUE PATTERN and CUE VALIDITY showed significant main effects, a significant interaction with each other and significant interactions with DECISION TIME VS. CONFIDENCE. $^{7}$ Thus, in line with our expectations, the factors influenced decision time and confidence in opposing directions. Univariate analyses of decision times and confidence judgments were run to further explore these effects.

Decision Times. The median of decision times was 3.71 seconds, indicating that individuals followed the time pressure instruction $(S D=4.29$, skew $=8.26$, kurtosis $=127.7)$. Note that the increase in decision times as compared to the previous experiments is likely because six rather than three cues were provided and that, unlike in Experiments 1 and 2, the presentation order of cues was randomized. For the further analyses, decision times were again logtransformed to reduce the influence of outliers and to account for deviations from normal distribution. We conducted a 6 (CUE PATTERN) x 4 (CUE VALIDITY) x 3 (REPETITION) repeated measurement ANOVA with log-transformed decision times as dependent variables. The main effects for CUE PATTERN turned out to be significant, $F(2.8,170.8)=56.8, p<$ $.001, \eta^{2}=.48$. The longest decision times were observed for cue pattern 5 and the lowest deci-

The omnibus tests revealed significant main effects for the factors CUE PATTERN, Pillais $V=.67, F(5$, $58)=28.9, p<.001, \eta 2=.67$, CUE VALIDITY, Pillais $V=.46, F(3,60)=16.8, p<.001, \eta 2=.46$, and DECISION TIME VS. CONFIDENCE, Pillais $V=.67, F(1,62)=125.3, p<.001, \eta 2=.67$. Furthermore, the following two-way interactions were significant: DECISION TIME VS. CONFIDENCE by CUE VALIDITY, Pillais $V=.46, F(3,60)=17.1, p<.001, \eta 2=.46$, DECISION TIME VS. CONFIDENCE by CUE PATTERN, Pillais $V=.67, F(5,58)=23.9, p<.001, \eta 2=.67$, and CUE VALIDITY by CUE PATTERN, Pillais $V=.49, F(15,48)=3.1, p<.01, \eta 2=.49$. Moreover we obtained a significant three-way interaction between DECISION TIME VS. CONFIDENCE, CUE VALIDITY, and CUE PATTERN, Pillais $V=.50, F(15,48)=3.1, p<.01, \eta 2=.50$. 
sion times were found for cue patterns 1 and 2 (Figure 6; SE ranged from 0.014 to 0.027). Thus, the finding that decision times are particularly long in decisions with conflicting cue information (i.e., cue patterns 3, 4, and 5) could be replicated. There was also a significant main effect for CUE VALIDITY, $F(2.6,163.3)=22.5, p<.001, \eta^{2}=.27$. Decision times decreased with increasing validity of cue 1 (see Figure 6). In addition, there was a significant main effect for REPETITION, indicating learning effects, $F(1.4,84.6)=128.1, p<.001, \eta^{2}=$ .67. The means of the log-transformed decision times for the three repetitions (with $S E$ in parentheses) were 3.68 (0.016), 3.59 (0.012), and 3.54 (0.012). The interaction between CUE VALIDITY and CUE PATTERN also turned out to be significant, $F(10.1,623.3)=3.1, p<$ $.01, \eta^{2}=.05$.

Figure 6

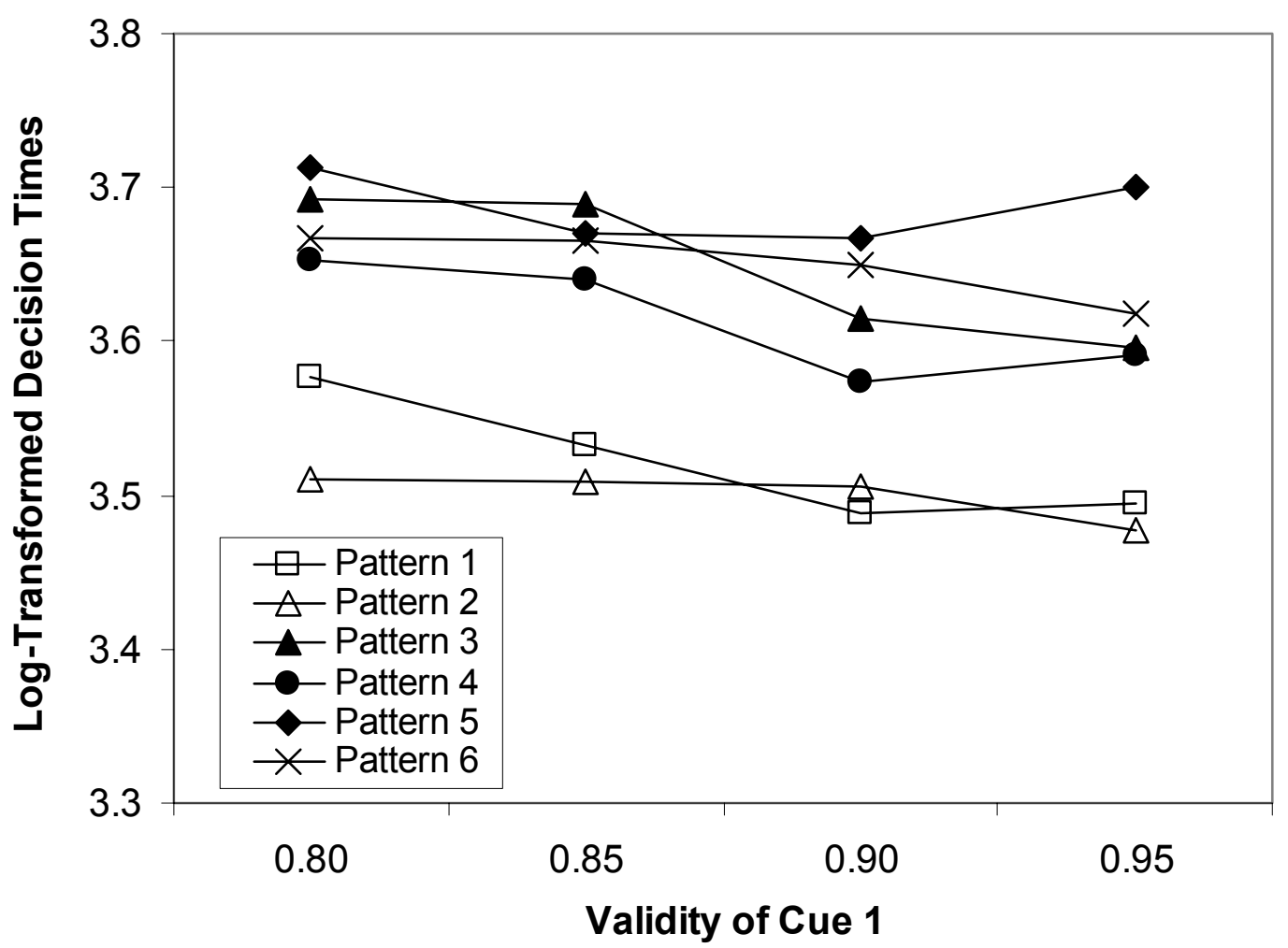

Figure 6. Log-transformed decision times in Experiment 3.

To explore whether decision times differ between LEX/TTB users and WADD users, we added another factor to the ANOVA. Specifically, we considered the result of the individual strategy classification as an additional factor. In this 4-factorial ANOVA, the factor DECISION STRATEGY had no main effect on decision time, $F(1,61)=0.36, p=.55, \eta^{2}=.01$. Both the two-way interaction between DECISION STRATEGY and CUE VALIDITY and the three-way interaction between DECISION STRATEGY, CUE VALIDITY, and CUE PATTERN turned out to be significant, $F(2.6,160.2)=3.0, p<.05, \eta^{2}=.05, F(10.1,617.4)=2.6$, $p<.01, \eta^{2}=.04$. Given the null effect of DECISION STRATEGY, we can conclude that participants were able to integrate all pieces of evidence according to a WADD rule in approxi- 
mately the same time that (other) participants needed to apply a simple LEX/TTB rule. However, the substantial interactions indicate that different decision strategies led to different patterns of decision time (cf. Table 1), although it should be noted that the effect sizes for these interactions are relatively low. WADD users showed patterns that could mainly be explained by the application of $\mathrm{WADD}_{\text {auto, }}$, whereas LEX/TTB users showed considerable differences in decision times - a finding that could hardly be explained by LEX/TTB. This could indicate that a considerable proportion of WADD users were still misclassified as LEX/TTB users (see Footnote 3).

Confidence Judgments. Confidence judgments were analyzed using a 6 (CUE PATTERN) x 4 (CUE VALIDITY) x 3 (REPETITION) repeated measurement ANOVA. The CUE PATTERN produced a significant main effect, $F(2.5,152.4)=48.4, p<.001, \eta^{2}=.44$. Confidence ratings were higher for cue patterns 1,2, and 6 than for the remaining cue patterns (Figure 7; $S E$ ranged from 3.2 to 6.0). We also obtained a significant main effect for CUE VALIDITY, $F(2.0,125.0)=34.7, p<.001, \eta^{2}=.36$. Confidence increased with increasing validity of cue 1 (see Figure 7). There was a significant interaction between CUE VALIDITY and CUE PATTERN, $F(8.3,516.1)=7.3, p<.001, \eta^{2}=.11$. The cue validity manipulation led to a general increase in confidence in all cue patterns except cue pattern 5 , in which a decrease was observed.

Figure 7

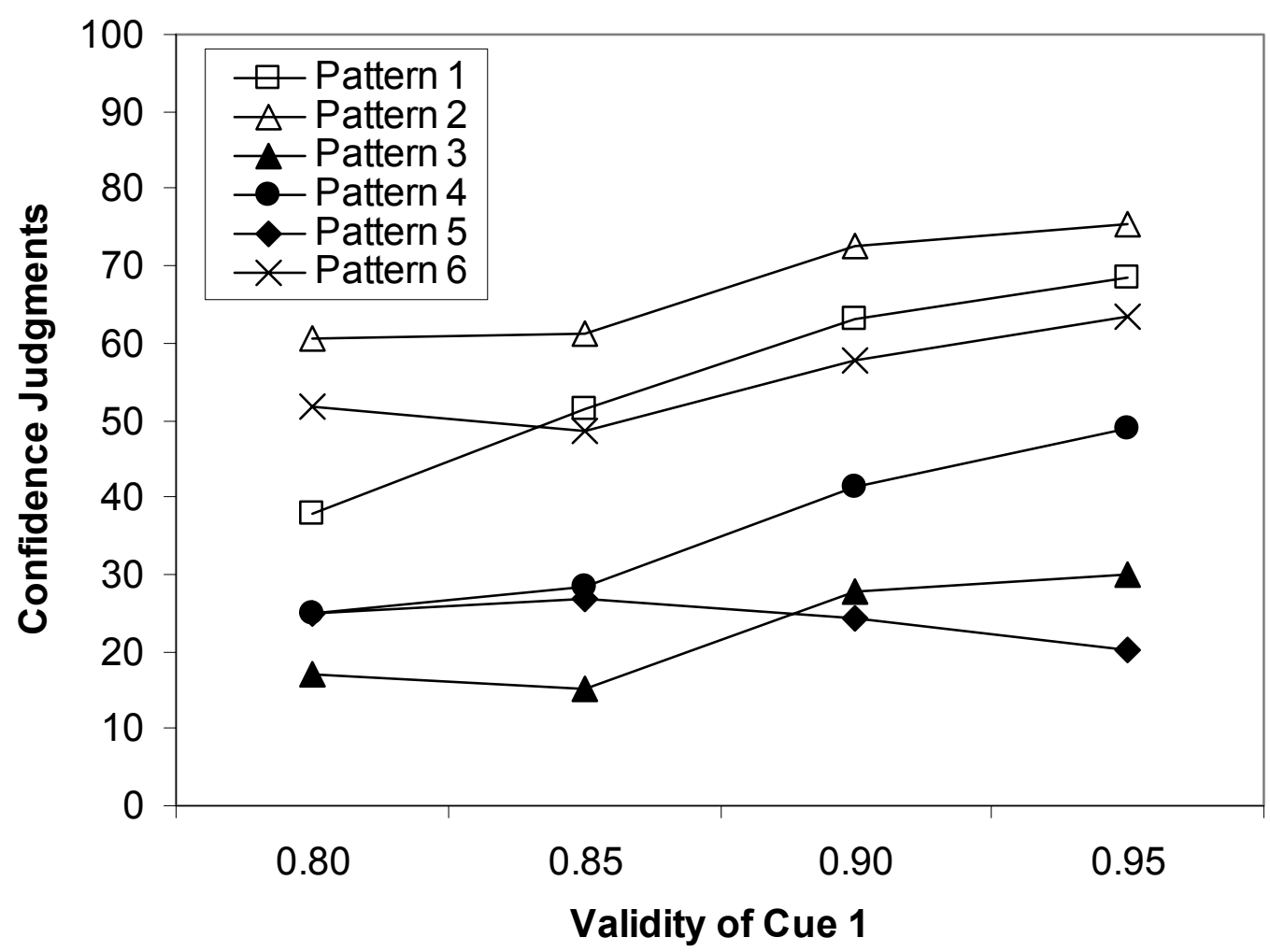

Figure 7. Confidence judgments in Experiment 3 with high values indicating a high level of confidence. Ratings could range from -100 to 100. 
To test more specifically the hypothesis that confidence judgments increase with increasing differences between the weighted cue values of options, we computed correlations between difference scores $D$ (i.e., difference between total evidence for options) and confidence judgments. Difference scores were computed by $D=\left|\sum c_{i}{ }^{O_{1}} w_{i}{ }^{O_{1}}-\sum c_{i}{ }^{O_{2}} w_{i}{ }^{O_{2}}\right|$ in which $c_{i}^{O_{1}}$ and $c_{i}{ }^{O_{2}}$ are cue values of cue $i$ for options $\mathrm{A}$ and $\mathrm{B}$ (i.e., -1 or 1 ); $w_{i}^{{ }^{O_{1}}}$ and $w_{i}{ }^{O_{2}}$ are decision weights for options A and B. Decision weights were calculated in two different ways. First, according to an ignorant WADD strategy, accuracy probabilities (e.g., .75 for cue 2) were directly used as decision weights $\left(w_{\text {cue }}=p_{\text {cue }}\right)$; second, difference scores were calculated by correcting for the fact that cues with an accuracy probability of .50 only $\left(w_{\text {cue }}=p_{\text {cue }}-.50\right)$ are uninformative. Correlations were calculated between confidence judgments and difference scores $D$ based on ignorant WADD and corrected WADD. There was a significantly negative correlation for difference scores based on ignorant WADD, $r=-.51, t(70)=-5.01, p<.001$ (two-tailed), but a significantly positive correlation for corrected difference scores, $r=.35$, $t(70)=3.16, p=.002$ (two-tailed). We did not expect a positive correlation for ignorant WADD because choice data already indicated that individuals corrected their decision weights. For the corrected difference scores, we obtained a substantial positive correlation. This supports the hypothesis derived from WADD strategies that the difference of the weighted cue values between options influences confidence judgments. Thus, the findings lend further support to our general hypothesis that individuals integrate information in a weighted additive manner. If cue validities or cue values were ignored, no correlation would be expected.

\section{Discussion}

In the third experiment, the general findings of the previous experiments could be replicated and strengthened by additional evidence. The majority of individuals took into account cue values and cue validities. Choices and confidence judgments indicate that the information was integrated in a weighted additive manner. The low overall decision times make it likely that individuals thereby relied on automatic processes; the systematic variations of decision time between cue patterns in line with the predictions of $\mathrm{WADD}_{\text {auto }}$ further corroborate this hypothesis. Note that the observed decision time of 3.7 seconds is not per se evidence for automatic processes. There are many cognitive tasks in which such a decision time would indicate the application of deliberate processes (e.g., simple Stroop tasks or categorization tasks as used in the implicit association test; Greenwald, McGhee, \& Schwarz, 1998). However, in decision tasks of the complexity used in our experiment, in which 12 cue values and 6 cue validities had to be considered, a decision time of 3.7 seconds substantially decreases the likelihood that $\mathrm{WADD}_{\text {del }}$ strategies were applied. ${ }^{8}$

8 As mentioned above, Lohse and Johnson (1996) estimated the time for applying such a strategy in an open Mouselab in decision tasks of similar complexity (i.e., 2 options and 7 attributes) to be 29.1 seconds. 
It could be shown that individuals are sensitive to minor manipulations of cue validities that do not change the general constellation of cue values. This clearly speaks against the idea that cue information is merely encoded as a constellation and compared with prototypes. Individuals integrate information in a weighted additive manner that is sensitive to minor changes in cue validities. Note that this observation also rules out alternative fast and frugal heuristics that are all based on ignorance or only ordinal considerations of cue validities (like the one discussed as an alternative explanation for the findings in Experiment 1). Finally, the findings cannot be explained by the recently proposed "complex heuristics" like TTB-CONFIGURAL (Garcia-Retamero et al., 2007) because, according to the heuristic, the minor manipulation of cue validities should not influence choices. Thus, our findings differ from that of Nosofsky and Bergert (2007), which found data in line with TTB-CONFIGURAL. It is highly likely that the many differences in materials and procedures (among other things, Nosofsky and Bergert used materials with interacting cue structures and provided feedback for decisions) might have caused the differences. Further research will be necessary to investigate the reasons for these differences more closely.

The analysis of confidence judgments lends additional support for $\mathrm{WADD}_{\text {auto }}$ strategies. In particular, the substantial correlation between confidence judgments and difference scores makes it unlikely that cue values or validities are ignored in the decision, although we cannot rule out that confidence judgments might have been based on other information than choices alone.

\section{General Discussion}

Process tracing studies have repeatedly shown that individuals employ simple strategies that minimize the amount of considered information and the mental effort invested in the decision (e.g., Payne et al., 1988). Although the question of strategy selection is still subject to theoretical debate (e.g., Glöckner \& Betsch, 2008; Lee \& Cummins, 2004; Newell, 2005; Rieskamp \& Otto, 2006), a few models converge in assuming that time and capacity constraints provoke strategy shifts towards a LEX/TTB rule (e.g., Bettman, Luce, \& Payne, 1998; Rieskamp \& Hoffrage, 1999). Moreover, joint evidence from simulations and empirical research suggests that LEX/TTB rules can lead to quite accurate decisions (Czerlinski, Gigerenzer, \& Goldstein, 1999), especially under time pressure (Payne et al., 1988). Taken together, these findings seem to corroborate the cornerstone assumptions of the bounded rationality approach that has been directing and inspiring psychological decision research over the last decades. One of these is that humans commonly lack the cognitive resources to apply extensional, compensatory strategies such as the WADD rule, particularly under time pressure. We hypothesized that this assumption does not hold if decision strategies are considered that capitalize on automatic processes for information integration and choice. When introducing the notion of bounded rationality, Herbert Simon (1955) already anticipated that the boundaries he described might only pertain to the deliberate side of the cognitive system: "My first empiri- 
cal proposition is that there is a complete lack of evidence that, in actual choice situations of any complexity, these [EU] computations can be, or are in fact, performed... but we cannot, of course, rule out the possibility that the unconscious is a better decision-maker than the conscious" (p. 104, italics added). Unfortunately, the research method regularly used in studying strategy application, Mouselab, hinders both the operation and the observation of automatic processes. It forces decision makers to engage in a step-by-step consideration of information, the units of observation being the steps represented by the movements of a computer mouse. The underlying assumption of this method is that overt information search behavior mimics hidden cognitive processes. We call this assumption into question, asserting instead that by directing individuals to uncover information one piece at a time, Mouselab binds task resources such that decision makers expend most of their time and effort on gathering information and thus cannot unfold their processing potential. Especially under time constraints, they are not able to collect as much information as they could process and therefore might work below their computational capacity. If this reasoning is true, the conclusions drawn from Mouselab studies would have to be reconsidered. Accordingly, the prevalence of shortcut strategies under Mouselab conditions might not provide evidence of limitations in the cognitive apparatus but simply show limitations in information search (i.e., uncovering hidden information in a matrix). Hence, we suspected that the predominance of LEX/TTB strategies under time constraints found in the majority of Mouselab studies was partially induced by the experimental procedure.

We tested this assumption in three experiments using choice-based strategy classification with open information presentation and compared the results with conditions using a standard Mouselab under different time pressure conditions. The results indicate that individuals are able to apply WADD rules within an astoundingly narrow time frame if information search is not restricted by environmental conditions. We were able to replicate prior findings in Experiment 2, where we employed the standard Mouselab program. In such an environment, participants searched for information in accordance with the LEX/TTB rule when time limits were too tight to inspect all pieces of information. In a third experiment, we investigated the cognitive processes that allowed individuals to quickly integrate information according to a WADD rule. Here we showed that choices were sensitive to even small changes in cue validities. By keeping the ordinal structure of the cue hierarchy constant in the latter experiment, we could rule out the explanation that individuals only encode constellations of information and compare them to prototypes, exemplars or use heuristics which are based on complex configural cues. Taken together, the studies provide evidence that the predominance of simple, noncompensatory strategies documented in Mouselab studies may well have been caused by the experimental method and not by cognitive limitations. As demonstrated, Mouselab is likely to induce the application of deliberate processes and thereby hinder the application of automatic processes. Consequently, the total cognitive capacity of human decision makers is substantially underestimated. Testing the potential of the human mind in Mouselab is like testing the power of a Ferrari's engine in a parking lot. Obviously, the Ferrari cannot unfold its 
powers in such a constrained environment. Rather, we need to run it on a speedway before making a verdict about its performance.

Our results indicate that individuals are capable of performing decision strategies involving complex information integration in an astonishingly short time period. Thus, the assumption that limitations of the cognitive capacity for information integration cause the application of simple serial strategies - as proposed by proponents of the bounded rationality approach - has to be revised. As anticipated by Herbert Simon, there may be another possibility for relieving humans from the burden of endless, complex mathematical computations. Evolution may have equipped humans with very powerful cognitive tools that capitalize on the automatic integration of information (Glöckner, in press). It has been shown that even sticklebacks select mating partners by a weighted consideration of multiple pieces of evidence (Künzler \& Bakker, 2001) and that monkeys react to stimuli by considering probabilities and values in a weighted additive way (Glimcher, Dorris, \& Bayer, 2005). Thus, from an evolutionary perspective, it seems rather unlikely that humans lack the cognitive capacity for such operations.

Our conclusions concerning the differential influence of time pressure on automatic and deliberate decision strategies converge nicely with recent findings by Beilock and DeCaro (2007), who investigated the influence of working memory capacity, time pressure and structure of the environment on individuals' strategies and performance in solving complex mathematical problems. Individuals' problem-solving strategies were measured by analyzing self-reports. Statements were categorized to indicate rule-based strategies or estimations based on previous associations with problem operands. The former are deliberate strategies, the latter (at least) contain what we call automatic strategies. In environments in which rule-based strategies lead to good performance, rule-based deciders showed a worse performance under high as compared to low time pressure, whereas the inverse effect was observed for persons who relied on more automatic strategies. Thus, time pressure hampers deliberate processes but does not necessarily have a negative effect on automatic processes.

According to our experiments, automatic strategies are likely to be used if a quick inspection of information is possible. At the same time, Glöckner and Hodges (2006) also found evidence for automatic strategies in experiments in which information had to be retrieved from memory (but see Bröder \& Schiffer, 2003a). However, further research will be needed to investigate the application of automatic strategies under different context conditions, including monetary multi-attributive decisions, which have often been used in classic Mouselab studies. Based on our data it is not possible to conclusively differentiate between the automatic processing models proposed by different authors (e.g., Beach \& Mitchell, 1996; Busemeyer \& Townsend, 1993; Dougherty et al., 1999). Nevertheless, only such models that predict choices based on weighted additive information integration and systematic differences in decision times and confidence judgments can account for our findings. Particularly, the results nicely fit predictions derived from connectionist models of decision making (Betsch, 2005; Thagard \& Millgram, 1995; Simon et al., 2004; Glöckner, 2006; Glöckner \& Betsch, 2008). They are 
also in line with some evidence accumulation models (e.g., Busemeyer \& Townsend, 1993; Usher \& McClelland, 2004).

Although Mouselab may not be an appropriate method for studying the limitations of the cognitive system, it has its undisputable merits as a tool for studying search processes in environments that only allow for serial and effortful acquisition of information. Such situations can of course be found under natural conditions as well. For instance, consider a first-year law student who checks legal cases by looking up relevant aspects piece by piece in the literature. Customers who intend to buy a product without any prior knowledge about it will probably behave in a similar way. For these situations, findings from Mouselab studies are likely to be valid. However, generalizations about results based on this specific research paradigm should be made with caution. Observations and case studies on decision making in natural setting (e.g., Klein, 1999) indicate that experienced decision makers are capable of considering a huge amount of information even under severe time constraints. For instance, experienced chess players (e.g., Ferrari, Didierjean, \& Marmeche, 2006) or experienced judges (Glöckner, in press) base their decisions on complex constellations of information rather than selectively focusing on single cues or reasons.

An important lesson to be learned from the multiple strategy approach is that properties of the decision task influence decision-making processes (e.g., Beach \& Mitchell, 1978; Payne et al., 1992). Taking the present findings, it is important to differentiate decision tasks in which information is instantly available from those in which it is not. If information is not instantly available, it has to be looked up in a serial manner. If task constraints obstruct the depth of information search (e.g., time pressure) it is reasonable to assume that decision makers will order their search by the importance of information and may apply simple, noncompensatory strategies for choice (e.g., TTB/LEX, cf. Experiment 2; Payne et al., 1988). Similarly, in situations in which expected gains from the information search are estimated to be lower than its costs, only the most important information may be inspected (cf. Beach \& Mitchell, 1978; Newell \& Shanks, 2003). In contrast, if the task enables relevant information to be accessed immediately, as it was the case in our open Mouselab, individuals are likely to employ complex strategies for choice (e.g., WADD ${ }_{\text {auto }}$ ), even under time constraints.

As already mentioned before, in several other studies, strategy shifts to noncompensatory strategies under time pressure were observed without relying on Mouselab procedure (for an overview, see Edland \& Svenson, 1993). Thus, we do not argue that all findings concerning strategy shifts under time pressure can be explained by information search constraints induced by Mouselab or other methods. However, according to our findings it should be acknowledged that humans' total cognitive capacity for information integration is higher than usually estimated.

To conclude, more research is needed that aims at exploring and disentangling the effects of the research method and other context variables. From our point of view, it is important to be aware of the limitations of a particular research method. Some methods (i.e., Mouselab; think- 
aloud protocols) appear unsuitable for identifying an important class of decision strategies, namely, strategies that capitalize on automatic processing of information. Relying more strongly on nonobtrusive methods of process tracing (e.g., eye tracking) and considering multiple correlates of internal processes (e.g., decision times, confidence judgments) might help to improve our understanding of automatic processes in decision making. 


\section{References}

Ariely, D, \& Zakay, D. (2001). A timely account of the role of duration in decision making. Acta Psychological, 108, 187-207.

Bargh, J. A., \& Chartrand, T. L. (1999). The unbearable automaticity of being. American Psychologist, 54, 462-479.

Bargh, J. A., \& Williams, E. L. (2006). The automaticity of social life. Current Directions in Psychological Research, 15, 2-4.

Beach, L. R., \& Mitchell, T. R. (1978). A contingency model for the selection of decision strategies. Academy of Management Review, 3, 439-449.

Beach, L. R., \& Mitchell, T. R. (1996). Image theory, the unifying perspective. In L. R. Beach (Ed.), Decision making in the workplace: A unified perspective (pp. 1-20). Mahwah, NJ: Lawrence Erlbaum.

Beach, L. R., \& Potter, R.E. (1992). The pre-choice screening of options. Acta Psychologica, $81,115-126$.

Bergert, F. B., \& Nosofsky, R. M. (2007). A response-time approach to comparing generalized rational and take-the-best models of decision making. Journal of Experimental Psychology: Learning, Memory, and Cognition, 33, 107-129.

Betsch, T. (2005). Preference theory: An affect-based approach to recurrent decision making. In T. Betsch \& S. Haberstroh (Eds.), The routines of decision making (pp. 39-66). Mahwah, NJ: Lawrence Erlbaum.

Betsch, T. (2007). The nature of intuition and its neglect in research on judgment and decision making. In H. Plessner, C. Betsch \& T. Betsch (Eds.), Intuition in judgment and decision making (pp. 3-22). Mahwah, NJ: Lawrence Erlbaum.

Bettman, J. R., Luce, M. F., \& Payne, J. W. (1998). Constructive consumer choices. The Journal of Consumer Research, 25, 187-217.

Billings, R. S., \& Marcus, S. A. (1983). Measures of compensatory and noncompensatory models of behaviour: Process tracing versus policy capturing. Organizational Behavior and Human Performance, 31, 331-352.

Böckenholt, U., \& Hynan, L. S. (1994). Caveats on a process-tracing measure and a remedy. Journal of Behavioral Decision Making, 7, 103-117.

Broadbent, D. E. (1971). Decision and stress. London: Academic Press. 
Bröder, A. (2000a). Assessing the empirical validity of the "take-the-best" heuristic as a model of human probabilistic inference. Journal of Experimental Psychology: Learning, Memory, and Cognition, 26, 1332-1346.

Bröder, A. (2000b). "Take The Best-Ignore The Rest”. Wann entscheiden Menschen begrenzt rational? [When do people make boundedly rational decisions?]. Lengerich, Germany: Pabst Science Publishers.

Bröder, A. (2003). Decision making with the "Adaptive Toolbox": Influence of environmental structure, intelligence, and working memory load. Journal of Experimental Psychology: Learning, Memory, and Cognition, 29, 611-625.

Bröder, A., \& Schiffer, S. (2003a). Take the best versus simultaneous feature matching: Probabilistic inferences from memory and effects of representation format. Journal of Experimental Psychology: General, 132, 277-293.

Bröder, A., \& Schiffer, S. (2003b). Bayesian strategy assessment in multi-attributive decision making. Journal of Behavioral Decision Making, 16, 193-213.

Bröder, A., \& Gaissmaier, W. (in press). Sequential processing of cues in memory-based multi-attribute decisions. Psychonomic Bulletin and Review.

Busemeyer, J. R., \& Townsend, J. T. (1993). Decision field theory: A dynamic cognitive approach to decision making in an uncertain environment. Psychological Review, 100, 432-459.

Cartwright, D. \& Festinger, L. (1943). A quantitative theory of decision. Psychological Review, 50, 595-621.

Chaiken, S., \& Trope, Y. (Eds.). (1999). Dual-process theories in social psychology. New York: Guilford Press.

Christensen-Szalanski, J. J. (1978). Problem solving strategies: A selection mechanism, some implications and some data. Organisational Behavior and Human Performance, 22, 307-323.

Czerlinski, J., Gigerenzer, G., \& Goldstein, D. G. (1999). How good are simple heuristics? In Simple heuristics that make us smart (pp. 97-118). New York: Oxford University Press.

Beilock, S. L., \& DeCaro, M. S. (2007). From poor performance to success under stress: Working memory, strategy selection, and mathematical problem solving under pressure. Journal of Experimental Psychology: Learning, Memory, and Cognition, 33, 983-998.

Doherty, M. E., \& Kurz, E. M. (1996). Social judgement theory. Thinking and Reasoning, 2, 109-140. 
Dougherty, M. R. P., Gettys, C. F., \& Ogden, E. E. (1999). MINERVA-DM: A memory process model for judgements of likelihood. Psychological Review, 106, 108-209.

Edland, A., \& Svenson, O. (1993). Judgment and decision making under time pressure: Studies and findings. In O. Svenson \& A. J. Maule (Eds.), Time pressure and stress in human judgment and decision making (pp. 27-40). New York: Plenum Press.

Epstein, S. (1990). Cognitive-experiential self-theory. In L. Pervin (Ed.), Handbook of personality: Theory and research (pp. 165-192). New York: Guilford.

Fazio, R. H. (1990). A practical guide to the use of response latency in social psychological research. In C. Hendrick \& M. S. Clark (Eds.), Research methods in personality and social psychology (pp. 74-97). Thousand Oaks, CA: Sage Publications.

Ferrari, V., Didierjean, A., \& Marmeche, E. (2006). Dynamic perception in chess. The Quarterly Journal of Experimental Psychology, 59, 397-410.

Festinger, L. (1943). Studies in decision: I. Decision-time, relative frequency of judgment and subjective confidence as related to physical stimulus difference. Journal of Experimental Psychology, 32, 291-306.

Fishburn, P. C. (1974). Lexicographic orders, utilities, and decision rules: A survey. Management Science, 20, 1442-1472.

Frederick, S. (2002). Automated choice heuristics. In D. Griffin, T. Gilovich \& D. Kahneman (Eds.), Heuristics and biases: The psychology of intuitive judgment (pp. 548-558). New York: Cambridge University Press.

Garcia-Retamero, R., Hoffrage, U., \& Dieckmann, A. (2007). When one cue is not enough: Combining fast and frugal heuristics with compound cue processing. The Quarterly Journal of Experimental Psychology, 60(9), 1197-1215.

Gigerenzer, G., Hoffrage, U., \& Kleinbölting, H. (1991). Probabilistic mental models: A Brunswikian theory of confidence. Psychological Review, 98, 506-528.

Gigerenzer, G., \& Hoffrage, U. (1995). How to improve Bayesian reasoning without instruction: Frequency formats. Psychological Review, 102, 684-704.

Gigerenzer, G., \& Goldstein, D. G. (1996). Reasoning the fast and frugal way: Models of bounded rationality. Psychological Review, 103, 650-669.

Gigerenzer, G., Todd, P. M., \& the ABC Group (1999). Simple heuristics that make us smart. New York: Oxford University Press. 
Gigerenzer, G. (2004). Fast and frugal heuristics: The tools of bounded rationality. In D. Koehler \& N. Harvey (Eds.), Handbook of judgment and decision making (pp. 62-88). Oxford, UK: Blackwell.

Glass, G. V., \& Hopkins, K. D. (1996). Statistical methods in education and psychology. Boston, MA: Allyn and Bacon.

Glimcher, P. W., Dorris, M. C., \& Bayer, H. M. (2005). Physiological utility theory and the neuroeconomics of choice. Games and Economic Behavior, 52, 213-256.

Glöckner, A. (2006). Automatische Prozesse bei Entscheidungen [Automatic processes in decision making]. Hamburg: Kovac.

Glöckner, A., \& Hodges, S. D. (2006). Strategy selection in memory based decisions: Simplifying fast and frugal heuristics versus weighted compensatory strategies based on automatic information integration. Manuscript submitted for publication.

Glöckner, A., \& Betsch, T. (in press). Do people make decisions under risk based on ignorance? An empirical test of the priority heuristic against cumulative prospect theory. Organizational Behavior and Human Decision Processes.

Glöckner, A. \& Betsch, T. (2008). Modeling option and strategy choices with connectionist networks: Towards an integrative model of automatic and deliberate decision making. Judgment and Decision Making, 3(3), 215-228.

Glöckner, A. (2007). Does intuition beat fast and frugal heuristics? A systematic empirical analysis. In H. Plessner, C. Betsch, and T. Betsch (Eds.), Intuition in judgment and decision making (pp. 309-325). Mahwah, NJ: Lawrence Erlbaum.

Glöckner, A. (in press). How evolution outwits bounded rationality: The efficient interaction of automatic and deliberate processes in decision making and implications for institutions. In C. Engel and W. Singer (Eds.), Better than conscious. FIAS Workshop Report. Cambridge, MA: MIT Press.

Greenwald, A. G., McGhee, D. E., \& Schwartz, J. L. K. (1998). Measuring individual differences in implicit cognition: the implicit association test. Journal of Personality and Social Psychology, 74, 1464-1480.

Hammond, K. R., Hamm, R. M., Grassia, J., \& Pearson, T. (1987). Direct comparison of the relative efficiency on intuitive and analytical cognition. IEEE Transactions on Systems, Man and Cybernetics, 17, 753-770.

Hasher, L., \& Zacks, R. T. (1984). Automatic processing of fundamental information: The case of frequency of occurrence. American Psychologist, 12, 1372-1388. 
Hintzman, D. L. (1988). Judgments of frequency and recognition memory in a multiple-trace memory model. Psychological Review, 95, 528-551.

Hoffman, P. J. (1960). The paramorphic representation of clinical judgment. Psychological Bulletin, 57, 116-131.

Hogarth, R. (2001). Educating intuition. Chicago: University of Chicago Press.

Holyoak, K. J., \& Simon, D. (1999). Bidirectional reasoning in decision making by constraint satisfaction. Journal of Experimental Psychology: General, 128, 3-31.

Johnson, E. J., Payne, J. W., Schkade, D. A., \& Bettman, J. R. (1986). Monitoring information processing and decisions: The Mouselab system. Unpublished manuscript, Center for Decision Studies, Fuqua School of Business, Duke University.

Juslin, P., Olsson, H., \& Olsson, A.-C. (2003). Exemplar effects in categorization and multiple-cue judgment. Journal of Experimental Psychology: General, 132, 133-156.

Kahneman, D., Slovic, P., \& Tversky, A. (Eds.) (1982). Judgement under uncertainty: Heuristics and biases. Cambridge, UK: Cambridge University Press.

Kahneman, D. \& Frederick, S. (2002). Representativeness revisited: Attribute substitution in intuitive judgment. In T. Gilovich, D. Griffin \& D. Kahneman (Eds.), Heuristics and biases: The psychology of intuitive judgment (pp. 49-81). New York: Cambridge University Press.

Klein, G. (1999). Applied decision making. In P. A. Hancock (Ed.), Human performance and ergonomics (pp. 87-107). San Diego, CA: Academic Press.

Künzler, R., \& Bakker, C. M. (2001). Female preferences for single and combined traits in computer animated stickleback males. Behavioral Ecology, 12, 681-685.

Lee, M. D., \& Cummins, T. D. R. (2004). Evidence accumulation in decision making: Unifying the "take the best" and the "rational" models. Psychonomic Bulletin \& Review, 11, 343-352.

Lieberman, M. D. (2000). Intuition: A social cognition neuroscience approach. Psychological Review, 126, 109-137.

Lohse, G. L., \& Johnson, E. J. (1996). A comparison of two process tracing methods for choice tasks. Organizational Behavior and Human Decision Processes, 68, 28-43.

Maule, A. J. (1994). A componential investigation of the relation between structural modelling and cognitive accounts of human judgement. Acta Psychologica, 87, 199-216. 
Montgomery, H., \& Svenson, O. (1983). A think-aloud study of dominance structuring in decision making. In R. Tietz (Ed.), Aspiration levels in bargaining and economic decision making (pp. 366-383). Berlin: Springer.

Newell, B. R., \& Shanks, D. R. (2003). Take the best or look at the rest? Factors influencing "One-Reason" decision making. Journal of Experimental Psychology: Learning, Memory, and Cognition, 29, 53-65.

Newell, B. R. (2005). Re-visions of rationality? Trends in Cognitive Science, 9, 11-15.

Nosofsky, R. M., \& Bergert, F. B. (2007). Limitations of exemplar models of multi-attribute probabilistic inference. Journal of Experimental Psychology: Learning, Memory, and Cognition, 33, 999-1019.

Olsson, A.-C., Enkvist, T., \& Juslin, P. (2006). Go with the flow: How to master a nonlinear multiple-cue judgment task. Journal of Experimental Psychology: Learning, Memory, and Cognition, 32, 1371-1384.

Payne, J. W., Bettman, J. R., \& Johnson, E. J. (1988). Adaptive strategy selection in decision making. Journal of Experimental Psychology: Learning, Memory, \& Cognition, 14, $534-552$.

Payne, J. W., Bettman, J. R., \& Johnson, E. J. (1992). Behavioral decision research: A constructive processing perspective. Annual Reviews of Psychology, 43, 87-131.

Rieskamp, J., \& Hoffrage, U. (1999). When do people use simple heuristics and how can we tell? In G. Gigerenzer, P. M. Todd \& the ABC Research Group, Simple heuristics that make us smart (pp. 141-167). New York: Oxford University Press.

Rieskamp, J., \& Otto, P. E. (2006). SSL: A theory of how people learn to select strategies. Journal of Experimental Psychology: General, 135, 207-236.

Russo, J. E., \& Dosher, B. A. (1983). Strategies for multiattribute binary choices. Journal of Experimental Psychology: Learning, Memory, and Cognition, 9, 676-696.

Schneider, W., \& Shiffrin, R. M. (1977). Controlled and automatic human information processing: I. Detection, search, and attention. Psychological Review, 84, 1-66.

Shiffrin, R. M., \& Schneider, W. (1977). Controlled and automatic human information processing: II. Perceptual learning, automatic attending, and a general theory. Psychological Review, 84, 127-190.

Simon, D., Snow, C. J., \& Read, S .J. (2004). The redux of cognitive consistency theories: Evidence judgments by constraint satisfaction. Journal of Personality and Social Psychology, 86, 814-837. 
Simon, H. A. (1955). A behavioral model of rational choice. The Quarterly Journal of Economics, 69, 99-118.

Sloman, S. A. (2002). Two systems of reasoning. In T. Gilovich, D. Griffin \& D. Kahneman (Eds.), Heuristics and biases: The psychology of intuitive judgment (pp. 379-396). New York: Cambridge University Press.

Sundstroem, G.A. (1987). Information search and decision making: the effects of Information display. Acta Psychologica, 65, 165-179.

Svenson, O. (1989). Eliciting and analysing verbal protocols in process studies of judgement and decision making. In H. Montgomery \& O. Svenson (Eds.), Process and structure in human decision making (pp. 65-81). New York: Wiley.

Thagard, P. \& Millgram, E. (1995). Inference to the best plan: A coherence theory of decision. In A. Ram \& D. B. Leake (Eds.), Goal-driven learning (pp. 439-454). Cambridge, MA: MIT Press.

Tversky, A. (1972). Elimination by aspect: A theory of choice. Psychological Review, 79, 281-299.

Usher, M., \& McClelland, J. L. (2004). Loss aversion and inhibition in dynamical models of multialternative choice. Psychological Review, 111, 757-769.

Wegner, D. (1994). Ironic processes of mental control. Psychological Review, 101, 34-52.

Zajonc, B. (1980). Feeling and thinking: Preferences need no inferences. American Psychologist, 35, 151-175.

Zakay, M. P. (1993). The impact of time perception processes on decision making under time stress. In O. Svenson \& A. J. Maule (Eds.), Time pressure and stress in human judgement and decision making (pp. 59-72). New York: Plenum Press. 


\section{Appendix A: Instructions used in Experiment 1}

Imagine that you are the head of a company that produces orange juice. You receive offers from different orange vendors and have to decide which vendor to select. You have three testers $\mathrm{A}, \mathrm{B}$, and $\mathrm{C}$, who check each vendor's oranges for their quality. They give you information about each vendor: "+" means the vendor's oranges are of good quality, "-" means the vendor's oranges are of poor quality. [pagebreak] You know from experience that the testers' information varies in reliability: Tester A's information is correct in 8 out of 10 cases, tester B's information is correct in 6 out of 10 cases, and tester C's information is correct in 5 out of 10 cases. [pagebreak] In the experiment you will be repeatedly presented with offers from three different vendors and information from the testers $\mathrm{A}, \mathrm{B}$, and $\mathrm{C}$ in the following format:

\section{Orange Vendors}
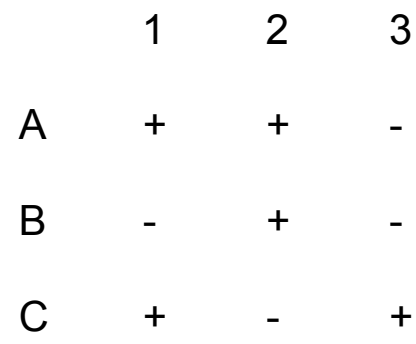

Your task is to select the vendor with the best-quality oranges. Please try to make good decisions and to proceed as quickly as possible. [pagebreak] Three keys are marked on the keyboard for use in selecting the vendors. Please lay three fingers of one hand on the three keys to avoid unnecessary errors. Hit the left key to select vendor 1, hit the middle key to select vendor 2 , and hit the right key to select vendor 3. 


\section{Appendix B: Additional Instructions used in Experiment 3}

\section{Explanations for the Information on Testers' Reliability}

The values for the reliability of the testers' predictions range from 50 percent to 100 percent. Fifty percent correct predictions mean that 5 of 10 of the tester's predictions are wrong. Because there are only two possible predictions (good-bad), this equates to random probability. This means that testers' whose predictions are 50 percent correct can be ignored because they provide no information about the quality of oranges. In contrast, the information of a tester who makes 100 percent correct predictions is always correct. The testers in the study will have different reliability values that are between 50 and 100 percent. 